\title{
Implicit Relaxed and Hybrid Methods with Regularization for Minimization Problems and Asymptotically Strict Pseudocontractive Mappings in the Intermediate Sense
}

\author{
Lu-Chuan Ceng, ${ }^{1}$ Qamrul Hasan Ansari, ${ }^{2,3}$ and Ching-Feng Wen ${ }^{4}$ \\ ${ }^{1}$ Department of Mathematics, Shanghai Normal University and Scientific Computing Key Laboratory of Shanghai Universities, \\ Shanghai 200234, China \\ ${ }^{2}$ Department of Mathematics, Aligarh Muslim University, Aligarh 202 002, India \\ ${ }^{3}$ Department of Mathematics and Statistics, King Fahd University of Petroleum \& Minerals, Dhahran, Saudi Arabia \\ ${ }^{4}$ Center for Fundamental Science, Kaohsiung Medical University, Kaohsiung 80708, Taiwan
}

Correspondence should be addressed to Ching-Feng Wen; cfwen@kmu.edu.tw

Received 1 February 2013; Accepted 11 March 2013

Academic Editor: Jen-Chih Yao

Copyright (C) 2013 Lu-Chuan Ceng et al. This is an open access article distributed under the Creative Commons Attribution License, which permits unrestricted use, distribution, and reproduction in any medium, provided the original work is properly cited.

We first introduce an implicit relaxed method with regularization for finding a common element of the set of fixed points of an asymptotically strict pseudocontractive mapping $S$ in the intermediate sense and the set of solutions of the minimization problem (MP) for a convex and continuously Frechet differentiable functional in the setting of Hilbert spaces. The implicit relaxed method with regularization is based on three well-known methods: the extragradient method, viscosity approximation method, and gradient projection algorithm with regularization. We derive a weak convergence theorem for two sequences generated by this method. On the other hand, we also prove a new strong convergence theorem by an implicit hybrid method with regularization for the MP and the mapping $S$. The implicit hybrid method with regularization is based on four well-known methods: the CQ method, extragradient method, viscosity approximation method, and gradient projection algorithm with regularization.

\section{Introduction}

In 1972, Goebel and Kirk [1] established that every asymptotically nonexpansive mapping $S: C \rightarrow C$ defined on a nonempty closed convex bounded subset of a uniformly convex Banach space, that is, there exists a sequence $\left\{k_{n}\right\}$ such that $\lim _{n \rightarrow \infty} k_{n}=1$ and

$$
\left\|S^{n} x-S^{n} y\right\| \leq k_{n}\|x-y\|, \quad \forall n \geq 1, \forall x, y \in C
$$

has a fixed point in $C$. It can be easily seen that every nonexpansive mapping is asymptotically nonexpansive, and every asymptotically nonexpansive mapping is uniformly Lipschitzian; that is, there exists a constant $\mathscr{L}>0$ such that

$$
\left\|S^{n} x-S^{n} y\right\| \leq \mathscr{L}\|x-y\|, \quad \forall n \geq 1, \forall x, y \in C .
$$

Several researchers have weaken the assumption on the mapping $S$. Bruck et al. [2] introduced the following concept of an asymptotically nonexpansive mapping in the intermediate sense.

Definition 1. Let $C$ be a nonempty subset of a normed space $X$. A mapping $S: C \rightarrow C$ is said to be asymptotically nonexpansive in the intermediate sense provided $S$ is uniformly continuous and

$$
\limsup _{n \rightarrow \infty} \sup _{x, y \in C}\left(\left\|S^{n} x-S^{n} y\right\|-\|x-y\|\right) \leq 0 .
$$

They also studied iterative methods for the approximation of fixed points of such mappings.

Recently, Kim and $\mathrm{Xu}$ [3] introduced the following concept of asymptotically $\kappa$-strict pseudocontractive mappings in setting of Hilbert spaces.

Definition 2. Let $C$ be a nonempty subset of a Hilbert space $H$. A mapping $S: C \rightarrow C$ is said to be an asymptotically $\kappa$ strict pseudocontractive mapping with sequence $\left\{\gamma_{n}\right\}$ if there 
exists a constant $\kappa \in[0,1)$ and a sequence $\left\{\gamma_{n}\right\}$ in $[0, \infty)$ with $\lim _{n \rightarrow \infty} \gamma_{n}=0$ such that

$$
\begin{aligned}
& \left\|S^{n} x-S^{n} y\right\|^{2} \\
& \leq\left(1+\gamma_{n}\right)\|x-y\|^{2}+\kappa\left\|x-S^{n} x-\left(y-S^{n} y\right)\right\|^{2} \\
& \forall n \geq 1, \quad \forall x, y \in C .
\end{aligned}
$$

They studied weak and strong convergence theorems for this class of mappings. It is important to note that every asymptotically $\kappa$-strict pseudocontractive mapping with sequence $\left\{\gamma_{n}\right\}$ is a uniformly $\mathscr{L}$-Lipschitzian mapping with $\mathscr{L}=$ $\sup \left\{\left(\kappa+\sqrt{1+(1-\kappa) \gamma_{n}}\right) /(1+\kappa): n \geq 1\right\}$

Very recently, Sahu et al. [4] considered the following concept of asymptotically $\kappa$-strict pseudocontractive mappings in the intermediate sense, which are not necessarily Lipschitzian.

Definition 3. Let $C$ be a nonempty subset of a Hilbert space $H$. A mapping $S: C \rightarrow C$ is said to be an asymptotically $\kappa$ strict pseudocontractive mapping in the intermediate sense with sequence $\left\{\gamma_{n}\right\}$ if there exist a constant $\kappa \in[0,1)$ and a sequence $\left\{\gamma_{n}\right\}$ in $[0, \infty)$ with $\lim _{n \rightarrow \infty} \gamma_{n}=0$ such that

$$
\begin{array}{r}
\limsup _{n \rightarrow \infty} \sup _{x, y \in C}\left(\left\|S^{n} x-S^{n} y\right\|^{2}-\left(1+\gamma_{n}\right)\|x-y\|^{2}\right. \\
\left.-\kappa\left\|x-S^{n} x-\left(y-S^{n} y\right)\right\|^{2}\right) \leq 0 .
\end{array}
$$

Let

$$
\begin{array}{r}
c_{n}:=\max \left\{0, \sup _{x, y \in C}\left(\left\|S^{n} x-S^{n} y\right\|^{2}-\left(1+\gamma_{n}\right)\|x-y\|^{2}\right.\right. \\
\left.\left.-\kappa\left\|x-S^{n} x-\left(y-S^{n} y\right)\right\|^{2}\right)\right\} .
\end{array}
$$

Then, $c_{n} \geq 0$ (for all $\left.n \geq 1\right), c_{n} \rightarrow 0(n \rightarrow \infty)$, and (5) reduces to the relation

$$
\begin{array}{r}
\left\|S^{n} x-S^{n} y\right\|^{2} \leq \\
+\kappa\left\|x-S^{n} x-\left(1+\gamma_{n}\right)\right\| x-y \|^{2} \\
\forall n \geq 1, \quad \forall x, y \in C .
\end{array}
$$

Whenever $c_{n}=0$ for all $n \geq 1$ in (7), then $S$ is an asymptotically $\kappa$-strict pseudocontractive mapping with sequence $\left\{\gamma_{n}\right\}$.

Let $f: C \rightarrow \mathbf{R}$ be a convex and continuously Fréchet differentiable functional. We consider the following minimization problem:

$$
\min _{x \in C} f(x)
$$

We assume that the minimization problem (8) has a solution, and the solution set of this problem is denoted by $\Gamma$.

To develop some new iterative methods for computing the approximate solutions of the minimization problem is one of the main areas of research in optimization and approximation theory. In the recent past, some study has also been done in the direction to suggest some iterative algorithms to compute the fixed point of a mapping which is also a solution of some minimization problem; for further detail, we refer to [5] and the references therein.

The main aim of this paper is to propose some iterative schemes for finding a common solution of fixed point set of an asymptotically $\kappa$-strict pseudocontractive mapping and the solution set of the minimization problem. In particular, we introduce an implicit relaxed algorithm with regularization for finding a common element of the fixed point set $\operatorname{Fix}(S)$ of an asymptotically $\kappa$-strict pseudocontractive mapping $S$ and the solution set $\Gamma$ of minimization problem (8). This implicit relaxed method with regularization is based on three well-known methods, namely, the extragradient method [6], viscosity approximation method, and gradient projection algorithm with regularization. We also propose an implicit hybrid algorithm with regularization for finding an element of $\operatorname{Fix}(S) \cap \Gamma$. The implicit hybrid method with regularization is based on four well-known methods, namely, the CQ method, extragradient method, viscosity approximation method, and gradient projection algorithm with regularization. The weak and strong convergence results of these two algorithms are established, respectively.

\section{Preliminaries}

Throughout the paper, unless otherwise specified, we use the following assumptions, notations, and terminologies.

We assume that $H$ is a real Hilbert space whose inner product and norm are denoted by $\langle\cdot, \cdot\rangle$ and $\|\cdot\|$, respectively, and $C$ is a nonempty closed convex subset of $H$. We write $x_{n} \rightarrow x$ to indicate that the sequence $\left\{x_{n}\right\}$ converges weakly to $x$ and $x_{n} \rightarrow x$ to indicate that the sequence $\left\{x_{n}\right\}$ converges strongly to $x$. Moreover, we use $\omega_{w}\left(x_{n}\right)$ to denote the weak $\omega$ limit set of the sequence $\left\{x_{n}\right\}$, that is,

$$
\begin{aligned}
& \omega_{w}\left(x_{n}\right):=\left\{x \in H: x_{n_{i}} \rightarrow x\right. \\
&\text { for some subsequence } \left.\left\{x_{n_{i}}\right\} \text { of }\left\{x_{n}\right\}\right\} .
\end{aligned}
$$

The metric (or nearest point) projection from $H$ onto $C$ is the mapping $P_{C}: H \rightarrow C$ which assigns to each point $x \in H$ the unique point $P_{C} x \in C$ satisfying the following property:

$$
\left\|x-P_{C} x\right\|=\inf _{y \in C}\|x-y\|=: d(x, C) .
$$

We mention some important properties of projections in the following proposition.

Proposition 4. For given $x \in H$ and $z \in C$,

(i) $z=P_{C} x \Leftrightarrow\langle x-z, y-z\rangle \leq 0$, for all $y \in C$;

(ii) $z=P_{C} x \Leftrightarrow\|x-z\|^{2} \leq\|x-y\|^{2}-\|y-z\|^{2}$, for all $y \in C$;

(iii) $\left\langle P_{C} x-P_{C} y, x-y\right\rangle \geq\left\|P_{C} x-P_{C} y\right\|^{2}$, for all $y \in H$.

Consequently, $P_{C}$ is nonexpansive.

Definition 5. A mapping $S: C \rightarrow H$ is said to be

(a) monotone if

$$
\langle S x-S y, x-y\rangle \geq 0, \quad \forall x, y \in C ;
$$


(b) $\eta$-strongly monotone if there exists a constant $\eta>0$ such that

$$
\langle S x-S y, x-y\rangle \geq \eta\|x-y\|^{2}, \quad \forall x, y \in C ;
$$

(c) $\alpha$-inverse-strongly monotone ( $\alpha$-ism) if there exists a constant $\alpha>0$ such that

$$
\langle S x-S y, x-y\rangle \geq \alpha\|S x-S y\|^{2}, \quad \forall x, y \in C .
$$

Obviously, if $S$ is $\alpha$-inverse-strongly monotone, then it is monotone and $(1 / \alpha)$-Lipschitz continuous. It can be easily seen that if $S: C \rightarrow C$ is nonexpansive, then $I-S$ is monotone. It is also easy to see that a projection mapping $P_{C}$ is 1 -ism. The inverse strongly monotone (also known as cocoercive) operators have been applied widely in solving practical problems in various fields.

We need some facts and tools which are listed in the form of the following lemmas.

Lemma 6. Let $X$ be a real inner product space. Then,

$$
\|x+y\|^{2} \leq\|x\|^{2}+2\langle y, x+y\rangle, \quad \forall x, y \in X .
$$

Lemma 7 (see [7, Proposition 2.4]). Let $\left\{x_{n}\right\}$ be a bounded sequence in a reflexive Banach space $X$. If $\omega_{w}\left(\left\{x_{n}\right\}\right)=\{x\}$, then $x_{n} \rightarrow x$.

Let $A: C \rightarrow H$ be a nonlinear mapping. The classical variational inequality problem (VIP) is to find $\bar{x} \in C$ such that

$$
\langle A \bar{x}, y-\bar{x}\rangle \geq 0, \quad \forall y \in C .
$$

The solution set of VIP is denoted by $\operatorname{VI}(C, A)$.

The theory of variational inequalities is a well-established subject in applied mathematics, nonlinear analysis, and optimization. For further details on variational inequalities, we refer to [8-13] and the references therein.

It is well known that the solution of a variational inequality can be characterized be a fixed point of a projection mapping. Therefore, by using Proposition 4(i), we have the following result.

Lemma 8. Let $A: C \rightarrow H$ be a monotone mapping. Then,

$$
u \in \mathrm{VI}(C, A) \Longleftrightarrow u=P_{C}(u-\lambda A u), \quad \text { for some } \lambda>0 \text {. }
$$

Lemma 9. The following assertions hold:

(a) $\|x-y\|^{2}=\|x\|^{2}-\|y\|^{2}-2\langle x-y, y\rangle$ for all $x, y \in H$;

(b) $\|\lambda x+\mu y+v z\|^{2}=\lambda\|x\|^{2}+\mu\|y\|^{2}+\nu\|z\|^{2}-$ $\lambda \mu\|x-y\|^{2}-\mu \nu\|y-z\|^{2}-\lambda\|x-z\|^{2}$ for all $x, y, z \in H$ and $\lambda, \mu, v \in[0,1]$ with $\lambda+\mu+\nu=1[14]$;

(c) if $\left\{x_{n}\right\}$ is a sequence in $H$ such that $x_{n} \rightarrow x$, then

$\limsup _{n \rightarrow \infty}\left\|x_{n}-y\right\|^{2}=\limsup _{n \rightarrow \infty}\left\|x_{n}-x\right\|^{2}+\|x-y\|^{2}, \quad \forall y \in H$.
Lemma 10 (see [4, Lemma 2.5]). For given points $x, y, z \in H$ and given also a real number $a \in \mathbf{R}$, the set

$$
\left\{v \in C:\|y-v\|^{2} \leq\|x-v\|^{2}+\langle z, v\rangle+a\right\}
$$

is convex (and closed).

Lemma 11 (see [4, Lemma 2.6]). Let $C$ be a nonempty subset of a Hilbert space $H$ and $S: C \rightarrow C$ an asymptotically $\kappa$-strict pseudocontractive mapping in the intermediate sense with sequence $\left\{\gamma_{n}\right\}$. Then,

$$
\begin{aligned}
& \left\|S^{n} x-S^{n} y\right\| \\
& \leq \frac{1}{1-\kappa}(\kappa\|x-y\| \\
& \left.\quad+\sqrt{\left(1+(1-\kappa) \gamma_{n}\right)\|x-y\|^{2}+(1-\kappa) c_{n}}\right)
\end{aligned}
$$

for all $x, y \in C$ and $n \geq 1$.

Lemma 12 (see [4, Lemma 2.7]). Let $C$ be a nonempty subset of a Hilbert space $H$ and $S: C \rightarrow C$ a uniformly continuous asymptotically $\kappa$-strict pseudocontractive mapping in the intermediate sense with sequence $\left\{\gamma_{n}\right\}$. Let $\left\{x_{n}\right\}$ be a sequence in $C$ such that $\left\|x_{n}-x_{n+1}\right\| \rightarrow 0$ and $\left\|x_{n}-S^{n} x_{n}\right\| \rightarrow 0$ as $n \rightarrow \infty$. Then, $\left\|x_{n}-S x_{n}\right\| \rightarrow 0$ as $n \rightarrow \infty$.

Lemma 13 (Demiclosedness Principle, [see [4, Proposition 3.1]]). Let $C$ be a nonempty closed convex subset of a Hilbert space $H$ and $S: C \rightarrow C$ be a continuous asymptotically $\kappa$-strict pseudocontractive mapping in the intermediate sense with sequence $\left\{\gamma_{n}\right\}$. Then $I-S$ is demiclosed at zero in the sense that if $\left\{x_{n}\right\}$ is a sequence in $C$ such that $x_{n} \rightarrow x \in C$ and $\lim \sup _{m \rightarrow \infty} \lim \sup _{n \rightarrow \infty}\left\|x_{n}-S^{m} x_{n}\right\|=0$, then $(I-S) x=0$.

Lemma 14 (see [4, Proposition 3.2]). Let $C$ be a nonempty closed convex subset of a Hilbert space $H$ and $S: C \rightarrow C$ a continuous asymptotically $\kappa$-strict pseudocontractive mapping in the intermediate sense with sequence $\left\{\gamma_{n}\right\}$ such that $\operatorname{Fix}(S) \neq \emptyset$. Then, $\operatorname{Fix}(S)$ is closed and convex.

To prove a weak convergence theorem by an implicit relaxed method with regularization for the minimization problem (8) and the fixed point problem of an asymptotically $\kappa$-strict pseudocontractive mapping in the intermediate sense, we need the following lemma due to Osilike et al. [15].

Lemma 15 (see [15, page 80]). Let $\left\{a_{n}\right\}_{n=1}^{\infty},\left\{b_{n}\right\}_{n=1}^{\infty}$, and $\left\{\delta_{n}\right\}_{n=1}^{\infty}$ be sequences of nonnegative real numbers satisfying the inequality

$$
a_{n+1} \leq\left(1+\delta_{n}\right) a_{n}+b_{n}, \quad \forall n \geq 1 .
$$

If $\sum_{n=1}^{\infty} \delta_{n}<\infty$ and $\sum_{n=1}^{\infty} b_{n}<\infty$, then $\lim _{n \rightarrow \infty} a_{n}$ exists. If, in addition, $\left\{a_{n}\right\}_{n=1}^{\infty}$ has a subsequence which converges to zero, then $\lim _{n \rightarrow \infty} a_{n}=0$. 
Corollary 16 (see [16, page 303]). Let $\left\{a_{n}\right\}_{n=0}^{\infty}$ and $\left\{b_{n}\right\}_{n=0}^{\infty}$ be two sequences of nonnegative real numbers satisfying the inequality

$$
a_{n+1} \leq a_{n}+b_{n}, \quad \forall n \geq 0 .
$$

If $\sum_{n=0}^{\infty} b_{n}$ converges, then $\lim _{n \rightarrow \infty} a_{n}$ exists.

Lemma 17 (see [17]). Every Hilbert space $H$ has the KadecKlee property; that is, given a sequence $\left\{x_{n}\right\} \subset H$ and a point $x \in H$, we have

$$
\left\|x_{n}\right\| \longrightarrow\|x\|, \quad x_{n} \rightarrow x \Longrightarrow x_{n} \longrightarrow x .
$$

It is well known that every Hilbert space $H$ satisfies Opial's condition [18]; that is, for any sequence $\left\{x_{n}\right\}$ with $x_{n} \rightarrow x$, we have

$$
\begin{array}{r}
\liminf _{n \rightarrow \infty}\left\|x_{n}-x\right\|<\liminf _{n \rightarrow \infty}\left\|x_{n}-y\right\|, \\
\forall y \in H \text { with } y \neq x .
\end{array}
$$

A set-valued mapping $T: H \rightarrow 2^{H}$ is called monotone if for all $x, y \in H, f \in T x$ and $g \in T y$ imply $\langle x-y, f-g\rangle \geq 0$. A monotone mapping $T: H \rightarrow 2^{H}$ is maximal if its graph $G(T)$ is not properly contained in the graph of any other monotone mapping. It is known that a monotone mapping $T$ is maximal if and only if for $(x, f) \in H \times H,\langle x-y, f-g\rangle \geq 0$ for all $(y, g) \in G(T)$ implies $f \in T x$. Let $A: C \rightarrow H$ be a monotone and $L$-Lipschitz continuous mapping, and let $N_{C} v$ be the normal cone to $C$ at $v \in C$, that is, $N_{C} v=\{w \in H$ : $\langle v-u, w\rangle \geq 0, \forall u \in C\}$. Define

$$
T v= \begin{cases}A v+N_{C} v, & \text { if } v \in C, \\ \emptyset, & \text { if } v \notin C .\end{cases}
$$

It is known that in this case $T$ is maximal monotone, and $0 \epsilon$ $T v$ if and only if $v \in \Omega$; see [19].

\section{Weak Convergence Theorem}

In this section, we will prove a weak convergence theorem for an implicit relaxed method with regularization for finding a common element of the set of fixed points of an asymptotically $\kappa$-strict pseudocontractive mapping $S$ : $C \rightarrow C$ in the intermediate sense and the set of solutions of the minimization problem (8) for a convex functional $f: C \rightarrow \mathbf{R}$ with $L$-Lipschitz continuous gradient $\nabla f$. This implicit relaxed method with regularization is based on the extragradient method, viscosity approximation method, and gradient projection algorithm (GPA) with regularization.

Theorem 18. Let $C$ be a nonempty bounded closed convex subset of a real Hilbert space $H$. Let $f: C \rightarrow \mathbf{R}$ be a convex functional with L-Lipschitz continuous gradient $\nabla f$, $Q: C \rightarrow C$ a $\rho$-contraction with coefficient $\rho \in[0,1)$, and $S: C \rightarrow C$ a uniformly continuous asymptotically $\kappa$ strict pseudocontractive mapping in the intermediate sense with sequence $\left\{\gamma_{n}\right\}$ such that $\operatorname{Fix}(S) \cap \Gamma$ is nonempty. Let $\left\{\gamma_{n}\right\}$ and $\left\{c_{n}\right\}$ be defined as in Definition 3. Let $\left\{x_{n}\right\}$ and $\left\{y_{n}\right\}$ be the sequences generated by

$$
\begin{gathered}
x_{1}=x \in C \text { chosen arbitrary, } \\
y_{n}=P_{C}\left(x_{n}-\lambda_{n} \mu_{n} \nabla f_{\alpha_{n}}\left(x_{n}\right)-\lambda_{n}\left(1-\mu_{n}\right) \nabla f_{\alpha_{n}}\left(y_{n}\right)\right), \\
t_{n}=P_{C}\left(x_{n}-\lambda_{n} \nabla f_{\alpha_{n}}\left(y_{n}\right)-\lambda_{n}\left(1-\mu_{n}\right) \nabla f_{\alpha_{n}}\left(t_{n}\right)\right), \\
x_{n+1}=\left(1-\sigma_{n}-\beta_{n}\right) t_{n}+\sigma_{n} Q t_{n}+\beta_{n} S^{n} t_{n}, \quad \forall n \geq 1,
\end{gathered}
$$

where $\left\{\alpha_{n}\right\}$ is a sequence $(0, \infty),\left\{\lambda_{n}\right\},\left\{\mu_{n}\right\}$ are sequences in $(0,1]$, and $\left\{\sigma_{n}\right\},\left\{\beta_{n}\right\}$ are sequences in $[0,1]$ satisfying the following conditions:

(i) $\delta \leq \beta_{n}$ and $\sigma_{n}+\beta_{n} \leq 1-\kappa-\tau$, for all $n \geq 1$ for some $\delta, \tau \in(0,1)$;

(ii) $\sum_{n=1}^{\infty} \alpha_{n}<\infty, \sum_{n=1}^{\infty} \gamma_{n}<\infty, \sum_{n=1}^{\infty} \sigma_{n}<\infty$ and $\sum_{n=1}^{\infty} c_{n}<\infty$

(iii) $\lim _{n \rightarrow \infty} \mu_{n}=1$;

(iv) $\lambda_{n}\left(\alpha_{n}+L\right)<1$, for all $n \geq 1$ and $\left\{\lambda_{n}\right\} \subset[a, b]$ for some $a, b \in(0,(1 / L))$.

Then, the sequences $\left\{x_{n}\right\},\left\{y_{n}\right\}$ converge weakly to some point $u \in \operatorname{Fix}(S) \cap \Gamma$.

Remark 19. Observe that for all $x, y \in C$ and all $n \geq 1$

$$
\begin{aligned}
& \| P_{C}\left(x_{n}-\lambda_{n} \mu_{n} \nabla f_{\alpha_{n}}\left(x_{n}\right)-\lambda_{n}\left(1-\mu_{n}\right) \nabla f_{\alpha_{n}}(x)\right) \\
& -P_{C}\left(x_{n}-\lambda_{n} \mu_{n} \nabla f_{\alpha_{n}}\left(x_{n}\right)-\lambda_{n}\left(1-\mu_{n}\right) \nabla f_{\alpha_{n}}(y)\right) \| \\
& \leq \|\left(x_{n}-\lambda_{n} \mu_{n} \nabla f_{\alpha_{n}}\left(x_{n}\right)-\lambda_{n}\left(1-\mu_{n}\right) \nabla f_{\alpha_{n}}(x)\right) \\
& \quad-\left(x_{n}-\lambda_{n} \mu_{n} \nabla f_{\alpha_{n}}\left(x_{n}\right)-\lambda_{n}\left(1-\mu_{n}\right) \nabla f_{\alpha_{n}}(y)\right) \| \\
& =\lambda_{n}\left(1-\mu_{n}\right)\left\|\nabla f_{\alpha_{n}}(x)-\nabla f_{\alpha_{n}}(y)\right\| \\
& \leq \lambda_{n}\left(\alpha_{n}+L\right)\|x-y\| .
\end{aligned}
$$

By Banach contraction principle, we know that for each $n \geq 1$, there exists a unique $y_{n} \in C$ such that

$$
y_{n}=P_{C}\left(x_{n}-\lambda_{n} \mu_{n} \nabla f_{\alpha_{n}}\left(x_{n}\right)-\lambda_{n}\left(1-\mu_{n}\right) \nabla f_{\alpha_{n}}\left(y_{n}\right)\right) .
$$

Also, observe that for all $x, y \in C$ and all $n \geq 1$

$$
\begin{aligned}
& \| P_{C}\left(x_{n}-\lambda_{n} \nabla f_{\alpha_{n}}\left(y_{n}\right)-\lambda_{n}\left(1-\mu_{n}\right) \nabla f_{\alpha_{n}}(x)\right) \\
& -P_{C}\left(x_{n}-\lambda_{n} \nabla f_{\alpha_{n}}\left(y_{n}\right)-\lambda_{n}\left(1-\mu_{n}\right) \nabla f_{\alpha_{n}}(y)\right) \| \\
& \leq \|\left(x_{n}-\lambda_{n} \nabla f_{\alpha_{n}}\left(y_{n}\right)-\lambda_{n}\left(1-\mu_{n}\right) \nabla f_{\alpha_{n}}(x)\right) \\
& \quad-\left(x_{n}-\lambda_{n} \nabla f_{\alpha_{n}}\left(y_{n}\right)-\lambda_{n}\left(1-\mu_{n}\right) \nabla f_{\alpha_{n}}(y)\right) \| \\
& =\lambda_{n}\left(1-\mu_{n}\right)\left\|\nabla f_{\alpha_{n}}(x)-\nabla f_{\alpha_{n}}(y)\right\| \\
& \leq \lambda_{n}\left(\alpha_{n}+L\right)\|x-y\| .
\end{aligned}
$$


Utilizing Banach contraction principle, for each $n \geq 1$, there exists a unique $t_{n} \in C$ such that

$$
t_{n}=P_{C}\left(x_{n}-\lambda_{n} \nabla f_{\alpha_{n}}\left(y_{n}\right)-\lambda_{n}\left(1-\mu_{n}\right) \nabla f_{\alpha_{n}}\left(t_{n}\right)\right) \text {. }
$$

Proof of Theorem 18. Note that the $L$-Lipschitz continuity of the gradient $\nabla f$ implies that $\nabla f$ is $(1 / L)$-ism [20], that is,

$$
\langle\nabla f(x)-\nabla f(y), x-y\rangle \geq \frac{1}{L}\|\nabla f(x)-\nabla f(y)\|^{2},
$$

$\forall x, y \in C$.

Thus, $\nabla f$ is monotone and $L$-Lipschitz continuous.

We divide the rest of the proof into several steps.

Step 1. $\lim _{n \rightarrow \infty}\left\|x_{n}-u\right\|$ exists for each $u \in \operatorname{Fix}(S) \cap \Gamma$.

Indeed, note that $t_{n}=P_{C}\left(x_{n}-\lambda_{n} \nabla f_{\alpha_{n}}\left(y_{n}\right)-\lambda_{n}(1-\right.$ $\left.\left.\mu_{n}\right) \nabla f_{\alpha_{n}}\left(t_{n}\right)\right)$ for all $n \geq 1$. Take $u \in \operatorname{Fix}(S) \cap \Gamma$ arbitrarily. From Proposition 4(ii), monotonicity of $\nabla f$, and $u \in \Gamma$, we have

$$
\begin{aligned}
& \left\|t_{n}-u\right\|^{2} \\
& \leq\left\|\left(x_{n}-\lambda_{n} \nabla f_{\alpha_{n}}\left(y_{n}\right)-\lambda_{n}\left(1-\mu_{n}\right) \nabla f_{\alpha_{n}}\left(t_{n}\right)\right)-u\right\|^{2} \\
& -\left\|\left(x_{n}-\lambda_{n} \nabla f_{\alpha_{n}}\left(y_{n}\right)-\lambda_{n}\left(1-\mu_{n}\right) \nabla f_{\alpha_{n}}\left(t_{n}\right)\right)-t_{n}\right\|^{2} \\
& =\left\|x_{n}-\lambda_{n}\left(1-\mu_{n}\right) \nabla f_{\alpha_{n}}\left(t_{n}\right)-u\right\|^{2} \\
& -\left\|x_{n}-\lambda_{n}\left(1-\mu_{n}\right) \nabla f_{\alpha_{n}}\left(t_{n}\right)-t_{n}\right\|^{2} \\
& +2 \lambda_{n}\left\langle\nabla f_{\alpha_{n}}\left(y_{n}\right), u-t_{n}\right\rangle \\
& =\left\|x_{n}-\lambda_{n}\left(1-\mu_{n}\right) \nabla f_{\alpha_{n}}\left(t_{n}\right)-u\right\|^{2} \\
& -\left\|x_{n}-\lambda_{n}\left(1-\mu_{n}\right) \nabla f_{\alpha_{n}}\left(t_{n}\right)-t_{n}\right\|^{2} \\
& +2 \lambda_{n}\left(\left\langle\nabla f_{\alpha_{n}}\left(y_{n}\right), u-y_{n}\right\rangle+\left\langle\nabla f_{\alpha_{n}}\left(y_{n}\right), y_{n}-t_{n}\right\rangle\right) \\
& =\left\|x_{n}-\lambda_{n}\left(1-\mu_{n}\right) \nabla f_{\alpha_{n}}\left(t_{n}\right)-u\right\|^{2} \\
& -\left\|x_{n}-\lambda_{n}\left(1-\mu_{n}\right) \nabla f_{\alpha_{n}}\left(t_{n}\right)-t_{n}\right\|^{2} \\
& +2 \lambda_{n}\left(\left\langle\nabla f_{\alpha_{n}}\left(y_{n}\right)-\nabla f_{\alpha_{n}}(u), u-y_{n}\right\rangle\right. \\
& \left.+\left\langle\nabla f_{\alpha_{n}}(u), u-y_{n}\right\rangle+\left\langle\nabla f_{\alpha_{n}}\left(y_{n}\right), y_{n}-t_{n}\right\rangle\right) \\
& \leq\left\|x_{n}-\lambda_{n}\left(1-\mu_{n}\right) \nabla f_{\alpha_{n}}\left(t_{n}\right)-u\right\|^{2} \\
& -\left\|x_{n}-\lambda_{n}\left(1-\mu_{n}\right) \nabla f_{\alpha_{n}}\left(t_{n}\right)-t_{n}\right\|^{2} \\
& +2 \lambda_{n}\left(\alpha_{n}\left\langle u, u-y_{n}\right\rangle+\left\langle\nabla f_{\alpha_{n}}\left(y_{n}\right), y_{n}-t_{n}\right\rangle\right) \\
& =\left\|x_{n}-u\right\|^{2}-\left\|x_{n}-t_{n}\right\|^{2} \\
& -2 \lambda_{n}\left(1-\mu_{n}\right)\left\langle\nabla f_{\alpha_{n}}\left(t_{n}\right), t_{n}-u\right\rangle \\
& +2 \lambda_{n}\left(\alpha_{n}\left\langle u, u-y_{n}\right\rangle+\left\langle\nabla f_{\alpha_{n}}\left(y_{n}\right), y_{n}-t_{n}\right\rangle\right)
\end{aligned}
$$

$$
\begin{aligned}
= & \left\|x_{n}-u\right\|^{2}-\left\|x_{n}-y_{n}\right\|^{2} \\
& -2\left\langle x_{n}-y_{n}, y_{n}-t_{n}\right\rangle-\left\|y_{n}-t_{n}\right\|^{2} \\
& +2 \lambda_{n}\left(\alpha_{n}\left\langle u, u-y_{n}\right\rangle+\left\langle\nabla f_{\alpha_{n}}\left(y_{n}\right), y_{n}-t_{n}\right\rangle\right) \\
& -2 \lambda_{n}\left(1-\mu_{n}\right) \\
& \times\left(\left\langle\nabla f_{\alpha_{n}}\left(t_{n}\right)-\nabla f_{\alpha_{n}}(u), t_{n}-u\right\rangle+\left\langle\nabla f_{\alpha_{n}}(u), t_{n}-u\right\rangle\right) \\
\leq & \left\|x_{n}-u\right\|^{2}-\left\|x_{n}-y_{n}\right\|^{2}-\left\|y_{n}-t_{n}\right\|^{2} \\
& +2\left\langle x_{n}-\lambda_{n} \nabla f_{\alpha_{n}}\left(y_{n}\right)-y_{n}, t_{n}-y_{n}\right\rangle \\
& +2 \lambda_{n} \alpha_{n}\left(\left\langle u, u-y_{n}\right\rangle+\left(1-\mu_{n}\right)\left\langle u, u-t_{n}\right\rangle\right) \\
\leq & \left\|x_{n}-u\right\|^{2}-\left\|x_{n}-y_{n}\right\|^{2}-\left\|y_{n}-t_{n}\right\|^{2} \\
& +2\left\langle x_{n}-\lambda_{n} \nabla f_{\alpha_{n}}\left(y_{n}\right)-y_{n}, t_{n}-y_{n}\right\rangle \\
& +2 \lambda_{n} \alpha_{n}\|u\|\left(\left\|y_{n}-u\right\|+\left\|t_{n}-u\right\|\right) .
\end{aligned}
$$

Since $y_{n}=P_{C}\left(x_{n}-\lambda_{n} \mu_{n} \nabla f_{\alpha_{n}}\left(x_{n}\right)-\lambda_{n}\left(1-\mu_{n}\right) \nabla f_{\alpha_{n}}\left(y_{n}\right)\right)$ and $\nabla f_{\alpha_{n}}$ is $\left(\alpha_{n}+L\right)$-Lipschitz continuous, by Proposition 4(i), we have

$$
\begin{aligned}
\left\langle x_{n}-\right. & \left.\lambda_{n} \nabla f_{\alpha_{n}}\left(y_{n}\right)-y_{n}, t_{n}-y_{n}\right\rangle \\
= & \left\langle x_{n}-\lambda_{n} \mu_{n} \nabla f_{\alpha_{n}}\left(x_{n}\right)-\lambda_{n}\left(1-\mu_{n}\right) \nabla f_{\alpha_{n}}\left(y_{n}\right)\right. \\
& \left.\quad-y_{n}, t_{n}-y_{n}\right\rangle \\
& +\lambda_{n} \mu_{n}\left\langle\nabla f_{\alpha_{n}}\left(x_{n}\right)-\nabla f_{\alpha_{n}}\left(y_{n}\right), t_{n}-y_{n}\right\rangle \\
\leq & \lambda_{n} \mu_{n}\left\langle\nabla f_{\alpha_{n}}\left(x_{n}\right)-\nabla f_{\alpha_{n}}\left(y_{n}\right), t_{n}-y_{n}\right\rangle \\
\leq & \lambda_{n} \mu_{n}\left\|\nabla f_{\alpha_{n}}\left(x_{n}\right)-\nabla f_{\alpha_{n}}\left(y_{n}\right)\right\|\left\|t_{n}-y_{n}\right\| \\
\leq & \lambda_{n}\left(\alpha_{n}+L\right)\left\|x_{n}-y_{n}\right\|\left\|t_{n}-y_{n}\right\| .
\end{aligned}
$$

So, we have

$$
\begin{aligned}
\left\|t_{n}-u\right\|^{2} \leq & \left\|x_{n}-u\right\|^{2}-\left\|x_{n}-y_{n}\right\|^{2}-\left\|y_{n}-t_{n}\right\|^{2} \\
& +2 \lambda_{n}\left(\alpha_{n}+L\right)\left\|x_{n}-y_{n}\right\|\left\|t_{n}-y_{n}\right\| \\
& +2 \lambda_{n} \alpha_{n}\|u\|\left(\left\|y_{n}-u\right\|+\left\|t_{n}-u\right\|\right) \\
\leq & \left\|x_{n}-u\right\|^{2}-\left\|x_{n}-y_{n}\right\|^{2}-\left\|y_{n}-t_{n}\right\|^{2} \\
& +\lambda_{n}^{2}\left(\alpha_{n}+L\right)^{2}\left\|x_{n}-y_{n}\right\|^{2}+\left\|t_{n}-y_{n}\right\|^{2} \\
& +2 \lambda_{n} \alpha_{n}\|u\|\left(\left\|y_{n}-u\right\|+\left\|t_{n}-u\right\|\right) \\
= & \left\|x_{n}-u\right\|^{2}+\left(\lambda_{n}^{2}\left(\alpha_{n}+L\right)^{2}-1\right)\left\|x_{n}-y_{n}\right\|^{2} \\
& +2 \lambda_{n} \alpha_{n}\|u\|\left(\left\|y_{n}-u\right\|+\left\|t_{n}-u\right\|\right) \\
\leq & \left\|x_{n}-u\right\|^{2}+2 \lambda_{n} \alpha_{n}\|u\|\left(\left\|y_{n}-u\right\|+\left\|t_{n}-u\right\|\right) .
\end{aligned}
$$


Therefore, from (33), $x_{n+1}=\left(1-\sigma_{n}-\beta_{n}\right) t_{n}+\sigma_{n} f\left(t_{n}\right)+\beta_{n} S^{n} t_{n}$ and $u=S u$. By Lemma 9(b), we have

$$
\begin{aligned}
\left\|x_{n+1}-u\right\|^{2} & \\
= & \left\|\left(1-\sigma_{n}-\beta_{n}\right)\left(t_{n}-u\right)+\sigma_{n}\left(Q t_{n}-u\right)+\beta_{n}\left(S^{n} t_{n}-u\right)\right\|^{2} \\
\leq & \left(1-\sigma_{n}-\beta_{n}\right)\left\|t_{n}-u\right\|^{2}+\sigma_{n}\left\|Q t_{n}-u\right\|^{2}+\beta_{n}\left\|S^{n} t_{n}-u\right\|^{2} \\
& -\left(1-\sigma_{n}-\beta_{n}\right) \beta_{n}\left\|t_{n}-S^{n} t_{n}\right\|^{2} \\
\leq & \left(1-\sigma_{n}-\beta_{n}\right)\left\|t_{n}-u\right\|^{2}+\sigma_{n}\left\|Q t_{n}-u\right\|^{2} \\
& +\beta_{n}\left[\left(1+\gamma_{n}\right)\left\|t_{n}-u\right\|^{2}+\kappa\left\|t_{n}-S^{n} t_{n}\right\|^{2}+c_{n}\right] \\
& -\left(1-\sigma_{n}-\beta_{n}\right) \beta_{n}\left\|t_{n}-S^{n} t_{n}\right\|^{2} \\
= & {\left[1-\sigma_{n}-\beta_{n}+\beta_{n}\left(1+\gamma_{n}\right)\right]\left\|t_{n}-u\right\|^{2}+\sigma_{n}\left\|Q t_{n}-u\right\|^{2} } \\
& +\beta_{n}\left(\kappa-\left(1-\sigma_{n}-\beta_{n}\right)\right)\left\|t_{n}-S^{n} t_{n}\right\|^{2}+\beta_{n} c_{n} \\
\leq & \left(1+\gamma_{n}\right)\left\|t_{n}-u\right\|^{2}+\sigma_{n}\left\|Q t_{n}-u\right\|^{2} \\
& +\beta_{n}\left(\kappa-\left(1-\sigma_{n}-\beta_{n}\right)\right)\left\|t_{n}-S^{n} t_{n}\right\|^{2}+c_{n} \\
\leq & \left(1+\gamma_{n}\right)\left\|t_{n}-u\right\|^{2}+\sigma_{n}\left\|Q t_{n}-u\right\|^{2}+c_{n} \\
\leq & \left(1+\gamma_{n}\right)\left[\left\|x_{n}-u\right\|^{2}+2 \lambda_{n} \alpha_{n}\|u\|\left(\left\|y_{n}-u\right\|+\left\|t_{n}-u\right\|\right)\right] \\
& +\sigma_{n}\left\|Q t_{n}-u\right\|^{2}+c_{n} \\
= & \left(1+\gamma_{n}\right)\left\|x_{n}-u\right\|^{2}+2 \alpha_{n} \lambda_{n}\left(1+\gamma_{n}\right)\|u\| \\
& \left(\left\|y_{n}-u\right\|+\left\|t_{n}-u\right\|\right)+\sigma_{n}\left\|Q t_{n}-u\right\|^{2}+c_{n} .
\end{aligned}
$$

Since $\sum_{n=1}^{\infty} \alpha_{n}<\infty, \sum_{n=1}^{\infty} \gamma_{n}<\infty, \sum_{n=1}^{\infty} \sigma_{n}<\infty$, and $\sum_{n=1}^{\infty} c_{n}<\infty$, from the boundedness of $C$, it follows that

$$
\begin{gathered}
\sum_{n=1}^{\infty}\left\{2 \alpha_{n} \lambda_{n}\left(1+\gamma_{n}\right)\|u\|\left(\left\|y_{n}-u\right\|+\left\|t_{n}-u\right\|\right)\right. \\
\left.+\sigma_{n}\left\|Q t_{n}-u\right\|^{2}+c_{n}\right\}<\infty .
\end{gathered}
$$

By Lemma 15, we have

$$
\lim _{n \rightarrow \infty}\left\|x_{n}-u\right\| \text { exists for all } u \in \operatorname{Fix}(S) \cap \Gamma \text {. }
$$

Step 2. $\lim _{n \rightarrow \infty}\left\|x_{n}-y_{n}\right\|=0$ and $\lim _{n \rightarrow \infty}\left\|x_{n}-t_{n}\right\|=0$. Indeed, substituting (33) in (34), we get

$$
\begin{aligned}
& \left\|x_{n+1}-u\right\|^{2} \\
& \leq\left(1+\gamma_{n}\right)\left\|t_{n}-u\right\|^{2}+\sigma_{n}\left\|Q t_{n}-u\right\|^{2}+c_{n} \\
& \leq\left(1+\gamma_{n}\right)\left[\left\|x_{n}-u\right\|^{2}+\left(\lambda_{n}^{2}\left(\alpha_{n}+L\right)^{2}-1\right)\left\|x_{n}-y_{n}\right\|^{2}\right. \\
& \left.+2 \lambda_{n} \alpha_{n}\|u\|\left(\left\|y_{n}-u\right\|+\left\|t_{n}-u\right\|\right)\right] \\
& +\sigma_{n}\left\|Q t_{n}-u\right\|^{2}+c_{n} \\
& =\left\|x_{n}-u\right\|^{2}+\gamma_{n}\left\|x_{n}-u\right\|^{2} \\
& +\left(1+\gamma_{n}\right)\left(\lambda_{n}^{2}\left(\alpha_{n}+L\right)^{2}-1\right)\left\|x_{n}-y_{n}\right\|^{2} \\
& +2 \alpha_{n} \lambda_{n}\left(1+\gamma_{n}\right)\|u\|\left(\left\|y_{n}-u\right\|+\left\|t_{n}-u\right\|\right) \\
& +\sigma_{n}\left\|Q t_{n}-u\right\|^{2}+c_{n},
\end{aligned}
$$

which hence implies that

$$
\begin{aligned}
(1+ & \left.\gamma_{n}\right)\left(b^{2}\left(\alpha_{n}+L\right)^{2}-1\right)\left\|x_{n}-y_{n}\right\|^{2} \\
\leq & \left(1+\gamma_{n}\right)\left(\lambda_{n}^{2}\left(\alpha_{n}+L\right)^{2}-1\right)\left\|x_{n}-y_{n}\right\|^{2} \\
\leq & \left\|x_{n}-u\right\|^{2}-\left\|x_{n+1}-u\right\|^{2}+\gamma_{n}\left\|x_{n}-u\right\|^{2} \\
& +2 \alpha_{n} \lambda_{n}\left(1+\gamma_{n}\right)\|u\|\left(\left\|y_{n}-u\right\|+\left\|t_{n}-u\right\|\right) \\
& +\sigma_{n}\left\|Q t_{n}-u\right\|^{2}+c_{n} .
\end{aligned}
$$

Since $\lim _{n \rightarrow \infty}\left\|x_{n}-u\right\|$ exists, $\alpha_{n} \rightarrow 0, \gamma_{n} \rightarrow 0, \sigma_{n} \rightarrow 0$, and $c_{n} \rightarrow 0$, from the boundedness of $C$, it follows that

$$
\lim _{n \rightarrow \infty}\left\|x_{n}-y_{n}\right\|=0
$$

Meantime, utilizing the arguments similar to those in (33), we have

$$
\begin{aligned}
\left\|t_{n}-u\right\|^{2} \leq & \left\|x_{n}-u\right\|^{2}-\left\|x_{n}-y_{n}\right\|^{2}-\left\|y_{n}-t_{n}\right\|^{2} \\
& +2 \lambda_{n}\left(\alpha_{n}+L\right)\left\|x_{n}-y_{n}\right\|\left\|t_{n}-y_{n}\right\| \\
& +2 \lambda_{n} \alpha_{n}\|u\|\left(\left\|y_{n}-u\right\|+\left\|t_{n}-u\right\|\right) \\
\leq & \left\|x_{n}-u\right\|^{2}-\left\|x_{n}-y_{n}\right\|^{2}-\left\|y_{n}-t_{n}\right\|^{2} \\
& +\lambda_{n}^{2}\left(\alpha_{n}+L\right)^{2}\left\|t_{n}-y_{n}\right\|^{2}+\left\|x_{n}-y_{n}\right\|^{2} \\
& +2 \lambda_{n} \alpha_{n}\|u\|\left(\left\|y_{n}-u\right\|+\left\|t_{n}-u\right\|\right) \\
= & \left\|x_{n}-u\right\|^{2}+\left(\lambda_{n}^{2}\left(\alpha_{n}+L\right)^{2}-1\right)\left\|t_{n}-y_{n}\right\|^{2} \\
& +2 \lambda_{n} \alpha_{n}\|u\|\left(\left\|y_{n}-u\right\|+\left\|t_{n}-u\right\|\right) .
\end{aligned}
$$


Substituting (40) in (34), we get

$$
\begin{aligned}
\| x_{n+1} & -u \|^{2} \\
\leq & \left(1+\gamma_{n}\right)\left\|t_{n}-u\right\|^{2}+\sigma_{n}\left\|Q t_{n}-u\right\|^{2}+c_{n} \\
\leq & \left(1+\gamma_{n}\right)\left[\left\|x_{n}-u\right\|^{2}+\left(\lambda_{n}^{2}\left(\alpha_{n}+L\right)^{2}-1\right)\left\|t_{n}-y_{n}\right\|^{2}\right. \\
& \left.+2 \lambda_{n} \alpha_{n}\|u\|\left(\left\|y_{n}-u\right\|+\left\|t_{n}-u\right\|\right)\right]+\sigma_{n}\left\|Q t_{n}-u\right\|^{2}+c_{n} \\
= & \left\|x_{n}-u\right\|^{2}+\gamma_{n}\left\|x_{n}-u\right\|^{2} \\
& +\left(1+\gamma_{n}\right)\left(\lambda_{n}^{2}\left(\alpha_{n}+L\right)^{2}-1\right)\left\|t_{n}-y_{n}\right\|^{2} \\
& +2 \alpha_{n} \lambda_{n}\left(1+\gamma_{n}\right)\|u\|\left(\left\|y_{n}-u\right\|+\left\|t_{n}-u\right\|\right) \\
& +\sigma_{n}\left\|Q t_{n}-u\right\|^{2}+c_{n},
\end{aligned}
$$

which hence implies that

$$
\begin{aligned}
(1+ & \left.\gamma_{n}\right)\left(b^{2}\left(\alpha_{n}+L\right)^{2}-1\right)\left\|t_{n}-y_{n}\right\|^{2} \\
\leq & \left(1+\gamma_{n}\right)\left(\lambda_{n}^{2}\left(\alpha_{n}+L\right)^{2}-1\right)\left\|t_{n}-y_{n}\right\|^{2} \\
\leq & \left\|x_{n}-u\right\|^{2}-\left\|x_{n+1}-u\right\|^{2}+\gamma_{n}\left\|x_{n}-u\right\|^{2} \\
& +2 \alpha_{n} \lambda_{n}\left(1+\gamma_{n}\right)\|u\|\left(\left\|y_{n}-u\right\|+\left\|t_{n}-u\right\|\right) \\
& +\sigma_{n}\left\|Q t_{n}-u\right\|^{2}+c_{n} .
\end{aligned}
$$

Since $\lim _{n \rightarrow \infty}\left\|x_{n}-u\right\|$ exists, $\alpha_{n} \rightarrow 0, \gamma_{n} \rightarrow 0, \sigma_{n} \rightarrow 0$, and $c_{n} \rightarrow 0$, from the boundedness of $C$ it follows that

$$
\lim _{n \rightarrow \infty}\left\|t_{n}-y_{n}\right\|=0 \text {. }
$$

This together with $\left\|x_{n}-y_{n}\right\| \rightarrow 0$ implies that

$$
\lim _{n \rightarrow \infty}\left\|x_{n}-t_{n}\right\|=0 \text {. }
$$

Step 3. $\lim _{n \rightarrow \infty}\left\|x_{n+1}-x_{n}\right\|=0$ and $\lim _{n \rightarrow \infty}\left\|x_{n}-S x_{n}\right\|=0$. Indeed, from (33) and (34), we conclude that

$$
\begin{aligned}
\| x_{n+1} & -u \|^{2} \\
\leq & \left(1+\gamma_{n}\right)\left\|t_{n}-u\right\|^{2}+\sigma_{n}\left\|Q t_{n}-u\right\|^{2} \\
& +\beta_{n}\left(\kappa-\left(1-\sigma_{n}-\beta_{n}\right)\right)\left\|t_{n}-S^{n} t_{n}\right\|^{2}+c_{n} \\
\leq & \left(1+\gamma_{n}\right)\left[\left\|x_{n}-u\right\|^{2}+2 \lambda_{n} \alpha_{n}\|u\|\left(\left\|y_{n}-u\right\|+\left\|t_{n}-u\right\|\right)\right] \\
& +\sigma_{n}\left\|Q t_{n}-u\right\|^{2} \\
& +\beta_{n}\left(\kappa-\left(1-\sigma_{n}-\beta_{n}\right)\right)\left\|t_{n}-S^{n} t_{n}\right\|^{2}+c_{n} \\
= & \left\|x_{n}-u\right\|^{2}+\gamma_{n}\left\|x_{n}-u\right\|^{2} \\
& +2 \alpha_{n} \lambda_{n}\left(1+\gamma_{n}\right)\|u\|\left(\left\|y_{n}-u\right\|+\left\|t_{n}-u\right\|\right) \\
& +\sigma_{n}\left\|Q t_{n}-u\right\|^{2}-\beta_{n}\left(\left(1-\sigma_{n}-\beta_{n}\right)-\kappa\right)\left\|t_{n}-S^{n} t_{n}\right\|^{2} \\
& +c_{n},
\end{aligned}
$$

which together with condition (i) yields

$$
\begin{aligned}
& \delta \tau\left\|t_{n}-S^{n} t_{n}\right\|^{2} \\
& \leq \beta_{n}\left(\left(1-\sigma_{n}-\beta_{n}\right)-\kappa\right)\left\|t_{n}-S^{n} t_{n}\right\|^{2} \\
& \leq\left\|x_{n}-u\right\|^{2}-\left\|x_{n+1}-u\right\|^{2}+\gamma_{n}\left\|x_{n}-u\right\|^{2} \\
&+2 \alpha_{n} \lambda_{n}\left(1+\gamma_{n}\right)\|u\|\left(\left\|y_{n}-u\right\|+\left\|t_{n}-u\right\|\right) \\
&+\sigma_{n}\left\|Q t_{n}-u\right\|^{2}+c_{n} .
\end{aligned}
$$

Since $\lim _{n \rightarrow \infty}\left\|x_{n}-u\right\|$ exists, $\alpha_{n} \rightarrow 0, \gamma_{n} \rightarrow 0, \sigma_{n} \rightarrow 0$, and $c_{n} \rightarrow 0$, from the boundedness of $C$ it follows that

$$
\lim _{n \rightarrow \infty}\left\|t_{n}-S^{n} t_{n}\right\|=0 .
$$

Note that

$$
\begin{aligned}
& \left\|x_{n+1}-x_{n}\right\| \\
& \quad \leq\left\|x_{n+1}-t_{n}\right\|+\left\|t_{n}-x_{n}\right\| \\
& \quad=\left\|\sigma_{n}\left(Q t_{n}-t_{n}\right)+\beta_{n}\left(S^{n} t_{n}-t_{n}\right)\right\|+\left\|t_{n}-x_{n}\right\| \\
& \quad \leq \sigma_{n}\left\|Q t_{n}-t_{n}\right\|+\left\|S^{n} t_{n}-t_{n}\right\|+\left\|t_{n}-x_{n}\right\| .
\end{aligned}
$$

From the boundedness of $C, \sigma_{n} \rightarrow 0,\left\|t_{n}-x_{n}\right\| \rightarrow 0$, and $\left\|S^{n} t_{n}-t_{n}\right\| \rightarrow 0$, we deduce that

$$
\lim _{n \rightarrow \infty}\left\|x_{n+1}-x_{n}\right\|=0
$$

Furthermore, observe that

$$
\left\|x_{n}-S^{n} x_{n}\right\| \leq\left\|x_{n}-t_{n}\right\|+\left\|t_{n}-S^{n} t_{n}\right\|+\left\|S^{n} t_{n}-S^{n} x_{n}\right\| .
$$

Utilizing Lemma 11, we have

$$
\begin{aligned}
& \left\|S^{n} t_{n}-S^{n} x_{n}\right\| \\
& \leq \frac{1}{1-\kappa} \\
& \quad \times\left(\kappa\left\|t_{n}-x_{n}\right\|\right. \\
& \left.\quad+\sqrt{\left(1+(1-\kappa) \gamma_{n}\right)\left\|t_{n}-x_{n}\right\|^{2}+(1-\kappa) c_{n}}\right)
\end{aligned}
$$

for every $n=1,2, \ldots$. Hence, it follows from $\left\|x_{n}-t_{n}\right\| \rightarrow 0$ that $\left\|S^{n} t_{n}-S^{n} x_{n}\right\| \rightarrow 0$. Thus, from (50) and $\left\|t_{n}-S^{n} t_{n}\right\| \rightarrow 0$, we get $\left\|x_{n}-S^{n} x_{n}\right\| \rightarrow 0$. Since $\left\|x_{n+1}-x_{n}\right\| \rightarrow 0$, $\| x_{n}-$ $S^{n} x_{n} \| \rightarrow 0$ as $n \rightarrow \infty$, and $S$ is uniformly continuous, we obtain from Lemma 12 that $\left\|x_{n}-S x_{n}\right\| \rightarrow 0$ as $n \rightarrow \infty$.

Step 4. $\omega_{w}\left(x_{n}\right) \subset \operatorname{Fix}(S) \cap \Gamma$.

Indeed, from the boundedness of $\left\{x_{n}\right\}$, we know that $\omega_{w}\left(\left\{x_{n}\right\}\right) \neq \emptyset$. Take $\widehat{u} \in \omega_{w}\left(\left\{x_{n}\right\}\right)$ arbitrarily. Then, there exists a subsequence $\left\{x_{n_{i}}\right\}$ of $\left\{x_{n}\right\}$ such that $\left\{x_{n_{i}}\right\}$ converges weakly to $\widehat{u}$. Note that $S$ is uniformly continuous and $\left\|x_{n}-S x_{n}\right\| \rightarrow 0$. 
Hence, it is easy to see that $\left\|x_{n}-S^{m} x_{n}\right\| \rightarrow 0$ for all $m \geq 1$. By Lemma 13, we obtain $\widehat{u} \in \operatorname{Fix}(S)$. Furthermore, we show $\widehat{u} \in \Gamma$. Since $x_{n}-t_{n} \rightarrow 0$ and $x_{n}-y_{n} \rightarrow 0$, we have $t_{n_{i}} \rightarrow \widehat{u}$ and $y_{n_{i}} \rightarrow \widehat{u}$. Let

$$
T v= \begin{cases}\nabla f(v)+N_{C} v, & \text { if } v \in C, \\ \emptyset, & \text { if } v \notin C,\end{cases}
$$

where $N_{C} v$ is the normal cone to $C$ at $v \in C$. We have already mentioned that in this case the mapping $T$ is maximal monotone, and $0 \in T v$ if and only if $v \in \operatorname{VI}(C, \nabla f)$; see [19] for more details. Let $G(T)$ be the graph of $T$ and let $(v, w) \in G(T)$. Then, we have $w \in T v=\nabla f(v)+N_{C} v$ and hence $w-\nabla f(v) \epsilon$ $N_{C} v$. So, we have $\langle v-t, w-\nabla f(v)\rangle \geq 0$ for all $t \in C$. On the other hand, from $t_{n}=P_{C}\left(x_{n}-\lambda_{n} \nabla f_{\alpha_{n}}\left(y_{n}\right)-\lambda_{n}\left(1-\mu_{n}\right) \nabla f_{\alpha_{n}}\left(t_{n}\right)\right)$ and $v \in C$, we have

$$
\left\langle x_{n}-\lambda_{n} \nabla f_{\alpha_{n}}\left(y_{n}\right)-\lambda_{n}\left(1-\mu_{n}\right) \nabla f_{\alpha_{n}}\left(t_{n}\right)-t_{n}, t_{n}-v\right\rangle \geq 0,
$$

and hence

$$
\left\langle v-t_{n}, \frac{t_{n}-x_{n}}{\lambda_{n}}+\nabla f_{\alpha_{n}}\left(y_{n}\right)+\left(1-\mu_{n}\right) \nabla f_{\alpha_{n}}\left(t_{n}\right)\right\rangle \geq 0 .
$$

Therefore, from $\langle v-t, w-\nabla f(v)\rangle \geq 0$ for all $t \in C$ and $t_{n_{i}} \in C$, we have

$$
\begin{aligned}
\langle v- & \left.t_{n_{i}}, w\right\rangle \\
\geq & \left\langle v-t_{n_{i}}, \nabla f(v)\right\rangle \\
\geq & \left\langle v-t_{n_{i}}, \nabla f(v)\right\rangle \\
& -\left\langle v-t_{n_{i}}, \frac{t_{n_{i}}-x_{n_{i}}}{\lambda_{n_{i}}}+\nabla f_{\alpha_{n_{i}}}\left(y_{n_{i}}\right)\right. \\
& \left.\quad+\left(1-\mu_{n_{i}}\right) \nabla f_{\alpha_{n_{i}}}\left(t_{n_{i}}\right)\right\rangle \\
= & \left\langle v-t_{n_{i}}, \nabla f(v)\right\rangle-\left\langle v-t_{n_{i}}, \frac{t_{n_{i}}-x_{n_{i}}}{\lambda_{n_{i}}}+\nabla f\left(y_{n_{i}}\right)\right\rangle \\
& -\alpha_{n_{i}}\left\langle v-t_{n_{i}}, y_{n_{i}}\right\rangle-\left(1-\mu_{n_{i}}\right)\left\langle v-t_{n_{i}}, \nabla f_{\alpha_{n_{i}}}\left(t_{n_{i}}\right)\right\rangle \\
= & \left\langle v-t_{n_{i}}, \nabla f(v)-\nabla f\left(t_{n_{i}}\right)\right\rangle \\
& +\left\langle v-t_{n_{i}}, \nabla f\left(t_{n_{i}}\right)-\nabla f\left(y_{n_{i}}\right)\right\rangle \\
& -\left(1-\mu_{n_{i}}\right)\left\langle v-t_{n_{i}}, \nabla f\left(t_{n_{i}}\right)-\nabla f\left(y_{\alpha_{n_{i}}}\left(t_{n_{i}}\right)\right\rangle-\left\langle v-t_{n_{i}}, \frac{t_{n_{i}}-x_{n_{i}}}{\lambda_{n_{i}}}\right\rangle\right. \\
& \left.-\left\langle v-t_{n_{i}}, \frac{t_{n_{i}}-x_{n_{i}}}{\lambda_{n_{i}}}\right\rangle-\alpha_{n_{n_{i}}}\right\rangle-\left(1-\mu_{n_{i}}\right)\left\langle v-t_{n_{i}}, \nabla f_{\alpha_{n_{i}}}\left(t_{n_{i}}\right)\right\rangle \\
& \left.\quad t_{n_{i}}, y_{n_{i}}\right\rangle
\end{aligned}
$$

Since $\left\|\nabla f\left(t_{n}\right)-\nabla f\left(y_{n}\right)\right\| \rightarrow 0$ (due to the Lipschitz continuity of $\nabla f),\left(t_{n}-x_{n}\right) /\left(\lambda_{n}\right) \rightarrow 0$ (due to $\left.\left\{\lambda_{n}\right\} \subset[a, b]\right), \alpha_{n} \rightarrow 0$, and $\mu_{n} \rightarrow 1$, we obtain $\langle v-\widehat{u}, w\rangle \geq 0$ as $i \rightarrow \infty$. Since $T$ is maximal monotone, we have $\widehat{u} \in T^{-1} 0$ and hence $\widehat{u} \in$ $\mathrm{VI}(C, \nabla f)$. Clearly, $\widehat{u} \in \Gamma$. Consequently, $\widehat{u} \in \operatorname{Fix}(S) \cap \Gamma$. This implies that $\omega_{w}\left(\left\{x_{n}\right\}\right) \subset \operatorname{Fix}(S) \cap \Gamma$.

Step 5. $\left\{x_{n}\right\}$ and $\left\{y_{n}\right\}$ converge weakly to the same point $u \in$ $\operatorname{Fix}(S) \cap \Gamma$.

Indeed, it is sufficient to show that $\omega_{w}\left(x_{n}\right)$ is a single-point set because $x_{n}-y_{n} \rightarrow 0$ as $n \rightarrow \infty$. Since $\omega_{w}\left(x_{n}\right) \neq \emptyset$, let us take two points $u, \widehat{u} \in \omega_{w}\left(x_{n}\right)$ arbitrarily. Then, there exist two subsequences $\left\{x_{n_{j}}\right\}$ and $\left\{x_{m_{k}}\right\}$ of $\left\{x_{n}\right\}$ such that $x_{n_{j}} \rightarrow u$ and $x_{m_{k}} \rightarrow \widehat{u}$, respectively. In terms of Step 4, we know that $u, \widehat{u} \in \operatorname{Fix}(S) \cap \Gamma$. Meantime, according to Step 1, we also know that there exist both $\lim _{n \rightarrow \infty}\left\|x_{n}-u\right\|$ and $\lim _{n \rightarrow \infty}\left\|x_{n}-\widehat{u}\right\|$. Let us show that $u=\widehat{u}$. Assume that $u \neq \widehat{u}$. From the Opial condition [18], it follows that

$$
\begin{aligned}
\lim _{n \rightarrow \infty}\left\|x_{n}-u\right\| & =\liminf _{j \rightarrow \infty}\left\|x_{n_{j}}-u\right\|<\liminf _{j \rightarrow \infty}\left\|x_{n_{j}}-\widehat{u}\right\| \\
& =\lim _{n \rightarrow \infty}\left\|x_{n}-\widehat{u}\right\|=\liminf _{k \rightarrow \infty}\left\|x_{m_{k}}-\widehat{u}\right\| \\
& <\liminf _{k \rightarrow \infty}\left\|x_{m_{k}}-u\right\|=\lim _{n \rightarrow \infty}\left\|x_{n}-u\right\| .
\end{aligned}
$$

This leads to a contradiction. Thus, we must have $u=\widehat{u}$. This implies that $\omega_{w}\left(x_{n}\right)$ is a single-point set. Without loss of generality, we may write $\omega_{w}\left(x_{n}\right)=\{u\}$. Consequently, by Lemma 7 , we obtain that $x_{n} \rightarrow u \in \operatorname{Fix}(S) \cap \Gamma$. Since $x_{n}-y_{n} \rightarrow 0$ as $n \rightarrow \infty$, we also have

$$
y_{n} \rightarrow u \in \operatorname{Fix}(S) \cap \Gamma .
$$

This completes the proof.

\section{Strong Convergence Theorem}

In this section, we establish a strong convergence theorem for an implicit hybrid method with regularization for finding a common element of the set of fixed points of an asymptotically $\kappa$-strict pseudocontractive mapping $S: C \rightarrow C$ in the intermediate sense and the set of solutions of the MP (8) for a convex functional $f: C \rightarrow \mathbf{R}$ with $L$-Lipschitz continuous gradient $\nabla f$. This implicit hybrid method with regularization is based on the CQ method, extragradient method, viscosity approximation method, and gradient projection algorithm (GPA) with regularization.

Theorem 20. Let $C$ be a nonempty closed convex subset of a real Hilbert space $H$. Let $f: C \rightarrow \mathbf{R}$ be a convex functional with L-Lipschitz continuous gradient $\nabla f, Q$ : $C \rightarrow C$ a $\rho$-contraction with coefficient $\rho \in[0,1)$, and $S: C \rightarrow C$ a uniformly continuous asymptotically $\kappa$-strict pseudocontractive mapping in the intermediate sense with sequence $\left\{\gamma_{n}\right\}$ such that $\operatorname{Fix}(S) \cap \Gamma$ is nonempty and bounded. 
Let $\left\{\gamma_{n}\right\}$ and $\left\{c_{n}\right\}$ be defined as in Definition 3. Let $\left\{x_{n}\right\},\left\{y_{n}\right\}$, and $\left\{z_{n}\right\}$ be the sequences generated by

$$
\begin{gathered}
x_{1}=x \in C \text { chosen arbitrary, } \\
y_{n}=P_{C}\left(x_{n}-\lambda_{n} \mu_{n} \nabla f_{\alpha_{n}}\left(x_{n}\right)-\lambda_{n}\left(1-\mu_{n}\right) \nabla f_{\alpha_{n}}\left(y_{n}\right)\right), \\
t_{n}=P_{C}\left(x_{n}-\lambda_{n} \nabla f_{\alpha_{n}}\left(y_{n}\right)-\lambda_{n}\left(1-\mu_{n}\right) \nabla f_{\alpha_{n}}\left(t_{n}\right)\right), \\
z_{n}=\left(1-\sigma_{n}-\beta_{n}\right) t_{n}+\sigma_{n} Q t_{n}+\beta_{n} S^{n} t_{n}, \\
C_{n}=\left\{z \in C:\left\|z_{n}-z\right\|^{2} \leq\left\|x_{n}-z\right\|^{2}+\theta_{n}\right\}, \\
Q_{n}=\left\{z \in C:\left\langle x_{n}-z, x-x_{n}\right\rangle \geq 0\right\}, \\
x_{n+1}=P_{C_{n} \cap Q_{n}} x, \quad \forall n \geq 1,
\end{gathered}
$$

where $\left\{\alpha_{n}\right\} \subset(0, \infty),\left\{\lambda_{n}\right\},\left\{\mu_{n}\right\} \subset(0,1],\left\{\sigma_{n}\right\},\left\{\beta_{n}\right\} \subset[0,1]$, $\theta_{n}=c_{n}+\left(\alpha_{n}+\gamma_{n}+\sigma_{n}\right) \Delta_{n}$ and

$$
\begin{gathered}
\Delta_{n}=\sup _{z \in \operatorname{Fix}(S) \cap \Gamma}\left\{\frac{33}{\mu_{n}^{4}}\left\|x_{n}-z\right\|^{2}+\left[\left((1-\rho)^{-1}+\gamma_{n}\right)^{2}+\frac{32}{\mu_{n}^{4}} \alpha_{n}^{2}\right]\right. \\
\left.\times\left(\|z\|^{2}+\|Q z-z\|^{2}\right)\right\}<\infty .
\end{gathered}
$$

Assume that the following conditions hold:

(i) $\delta \leq \beta_{n}$ and $\sigma_{n}+\beta_{n} \leq 1-\kappa$, for all $n \geq 1$ for some $\delta \in(0,1)$;

(ii) $\lim _{n \rightarrow \infty} \alpha_{n}=0$ and $\lim _{n \rightarrow \infty} \sigma_{n}=0$;

(iii) $\lim _{n \rightarrow \infty} \mu_{n}=1$;

(iv) $\lambda_{n}\left(\alpha_{n}+L\right)<1$, for all $n \geq 1$ and $\left\{\lambda_{n}\right\} \subset[a, b]$ for some $a, b \in(0,(1 / L))$.

Then, the sequences $\left\{x_{n}\right\},\left\{y_{n}\right\}$, and $\left\{z_{n}\right\}$ converge strongly to the same point $P_{\mathrm{Fix}(S) \cap \Gamma} x$.

Proof. Utilizing the condition $\lambda_{n}\left(\alpha_{n}+L\right)<1$ for all $n \geq 1$ and repeating the same arguments as in Remark 19, we can see that $\left\{y_{n}\right\}$ and $\left\{z_{n}\right\}$ are defined well. Note that the L-Lipschitz continuity of the gradient $\nabla f$ implies that $\nabla f$ is $(1 / L)$-ism [20], that is,

$$
\begin{array}{r}
\langle\nabla f(x)-\nabla f(y), x-y\rangle \geq \frac{1}{L}\|\nabla f(x)-\nabla f(y)\|^{2}, \\
\forall x, y \in C .
\end{array}
$$

Observe that

$$
\begin{aligned}
(\alpha+ & L)\left\langle\nabla f_{\alpha}(x)-\nabla f_{\alpha}(y), x-y\right\rangle \\
= & (\alpha+L)\left[\alpha\|x-y\|^{2}+\langle\nabla f(x)-\nabla f(y), x-y\rangle\right] \\
= & \alpha^{2}\|x-y\|^{2}+\alpha\langle\nabla f(x)-\nabla f(y), x-y\rangle+\alpha L\|x-y\|^{2} \\
& +L\langle\nabla f(x)-\nabla f(y), x-y\rangle \\
\geq & \alpha^{2}\|x-y\|^{2}+2 \alpha\langle\nabla f(x)-\nabla f(y), x-y\rangle \\
& +\|\nabla f(x)-\nabla f(y)\|^{2} \\
= & \|\alpha(x-y)+\nabla f(x)-\nabla f(y)\|^{2} \\
= & \left\|\nabla f_{\alpha}(x)-\nabla f_{\alpha}(y)\right\|^{2} .
\end{aligned}
$$

Hence, it follows that $\nabla f_{\alpha}=\alpha I+\nabla f$ is $(1 /(\alpha+L))$-ism. It is clear that $C_{n}$ is closed and $Q_{n}$ is closed and convex for every $n=1,2, \ldots$. As the defining inequality in $C_{n}$ is equivalent to the inequality

$$
\left\langle 2\left(x_{n}-z_{n}\right), z\right\rangle \leq\left\|x_{n}\right\|^{2}-\left\|z_{n}\right\|^{2}+\theta_{n}
$$

By Lemma 10, we also have that $C_{n}$ is convex for every $n=$ $1,2, \ldots$. As $Q_{n}=\left\{z \in C:\left\langle x_{n}-z, x-x_{n}\right\rangle \geq 0\right\}$, we have $\left\langle x_{n}-z, x-x_{n}\right\rangle \geq 0$ for all $z \in Q_{n}$, and by Proposition 4(i), we get $x_{n}=P_{\mathrm{Q}_{n}} x$.

We divide the rest of the proof into several steps.

Step 1. $\left\{x_{n}\right\},\left\{y_{n}\right\}$, and $\left\{z_{n}\right\}$ are bounded.

Indeed, take $u \in \operatorname{Fix}(S) \cap \Gamma$ arbitrarily. Taking into account $\lambda_{n}\left(\alpha_{n}+L\right)<1$, for all $n \geq 1$, we deduce that

$$
\begin{aligned}
& \left\|x_{n}-u-\lambda_{n} \mu_{n}\left(\nabla f_{\alpha_{n}}\left(x_{n}\right)-\nabla f_{\alpha_{n}}(u)\right)\right\|^{2} \\
& \leq\left\|x_{n}-u\right\|^{2}+\lambda_{n} \mu_{n}\left(\lambda_{n} \mu_{n}-\frac{2}{\alpha_{n}+L}\right)\left\|\nabla f_{\alpha_{n}}\left(x_{n}\right)-\nabla f_{\alpha_{n}}(u)\right\|^{2} \\
& \leq\left\|x_{n}-u\right\|^{2}+\lambda_{n}\left(\lambda_{n}-\frac{2}{\alpha_{n}+L}\right)\left\|\nabla f_{\alpha_{n}}\left(x_{n}\right)-\nabla f_{\alpha_{n}}(u)\right\|^{2} \\
& \leq\left\|x_{n}-u\right\|^{2} \\
& \left\|y_{n}-u\right\| \\
& =\| P_{C}\left(x_{n}-\lambda_{n} \mu_{n} \nabla f_{\alpha_{n}}\left(x_{n}\right)-\lambda_{n}\left(1-\mu_{n}\right) \nabla f_{\alpha_{n}}\left(y_{n}\right)\right) \\
& -P_{C}\left(u-\lambda_{n} \mu_{n} \nabla f(u)-\lambda_{n}\left(1-\mu_{n}\right) \nabla f(u)\right) \| \\
& \leq \|\left(x_{n}-\lambda_{n} \mu_{n} \nabla f_{\alpha_{n}}\left(x_{n}\right)-\lambda_{n}\left(1-\mu_{n}\right) \nabla f_{\alpha_{n}}\left(y_{n}\right)\right) \\
& -\left(u-\lambda_{n} \mu_{n} \nabla f(u)-\lambda_{n}\left(1-\mu_{n}\right) \nabla f(u)\right) \| \\
& \leq\left\|x_{n}-u-\lambda_{n} \mu_{n}\left(\nabla f_{\alpha_{n}}\left(x_{n}\right)-\nabla f(u)\right)\right\| \\
& +\lambda_{n}\left(1-\mu_{n}\right)\left\|\nabla f_{\alpha_{n}}\left(y_{n}\right)-\nabla f(u)\right\| \\
& \leq\left\|x_{n}-u-\lambda_{n} \mu_{n}\left(\nabla f_{\alpha_{n}}\left(x_{n}\right)-\nabla f_{\alpha_{n}}(u)\right)\right\| \\
& +\lambda_{n} \mu_{n}\left\|\nabla f_{\alpha_{n}}(u)-\nabla f(u)\right\|+\lambda_{n}\left(1-\mu_{n}\right) \\
& \times\left[\left\|\nabla f_{\alpha_{n}}\left(y_{n}\right)-\nabla f_{\alpha_{n}}(u)\right\|+\left\|\nabla f_{\alpha_{n}}(u)-\nabla f(u)\right\|\right] \\
& \leq\left\|x_{n}-u\right\|+\lambda_{n} \mu_{n} \alpha_{n}\|u\| \\
& +\lambda_{n}\left(1-\mu_{n}\right)\left[\left(\alpha_{n}+L\right)\left\|y_{n}-u\right\|+\alpha_{n}\|u\|\right] \\
& =\left\|x_{n}-u\right\|+\lambda_{n}\left(1-\mu_{n}\right)\left(\alpha_{n}+L\right)\left\|y_{n}-u\right\| \\
& +\lambda_{n} \alpha_{n}\|u\| \\
& \leq\left\|x_{n}-u\right\|+\left(1-\mu_{n}\right)\left\|y_{n}-u\right\|+\lambda_{n} \alpha_{n}\|u\|,
\end{aligned}
$$


which implies that

$$
\left\|y_{n}-u\right\| \leq \frac{1}{\mu_{n}}\left(\left\|x_{n}-u\right\|+\lambda_{n} \alpha_{n}\|u\|\right) .
$$

Meantime, we also have

$$
\begin{aligned}
& \left\|t_{n}-u\right\| \\
& =\| P_{C}\left(x_{n}-\lambda_{n} \nabla f_{\alpha_{n}}\left(y_{n}\right)-\lambda_{n}\left(1-\mu_{n}\right) \nabla f_{\alpha_{n}}\left(t_{n}\right)\right) \\
& -P_{C}\left(u-\lambda_{n} \nabla f(u)-\lambda_{n}\left(1-\mu_{n}\right) \nabla f(u)\right) \| \\
& \leq \|\left(x_{n}-\lambda_{n} \nabla f_{\alpha_{n}}\left(y_{n}\right)-\lambda_{n}\left(1-\mu_{n}\right) \nabla f_{\alpha_{n}}\left(t_{n}\right)\right) \\
& -\left(u-\lambda_{n} \nabla f(u)-\lambda_{n}\left(1-\mu_{n}\right) \nabla f(u)\right) \| \\
& \leq\left\|x_{n}-u\right\|+\lambda_{n}\left\|\nabla f_{\alpha_{n}}\left(y_{n}\right)-\nabla f(u)\right\| \\
& +\lambda_{n}\left(1-\mu_{n}\right)\left\|\nabla f_{\alpha_{n}}\left(t_{n}\right)-\nabla f(u)\right\| \\
& \leq\left\|x_{n}-u\right\| \\
& +\lambda_{n}\left[\left\|\nabla f_{\alpha_{n}}\left(y_{n}\right)-\nabla f_{\alpha_{n}}(u)\right\|+\left\|\nabla f_{\alpha_{n}}(u)-\nabla f(u)\right\|\right] \\
& +\lambda_{n}\left(1-\mu_{n}\right) \\
& \times\left[\left\|\nabla f_{\alpha_{n}}\left(t_{n}\right)-\nabla f_{\alpha_{n}}(u)\right\|+\left\|\nabla f_{\alpha_{n}}(u)-\nabla f(u)\right\|\right] \\
& \leq\left\|x_{n}-u\right\|+\lambda_{n}\left[\left(\alpha_{n}+L\right)\left\|y_{n}-u\right\|+\alpha_{n}\|u\|\right] \\
& +\lambda_{n}\left(1-\mu_{n}\right)\left[\left(\alpha_{n}+L\right)\left\|t_{n}-u\right\|+\alpha_{n}\|u\|\right] \\
& \leq\left\|x_{n}-u\right\|+\left\|y_{n}-u\right\|+\lambda_{n} \alpha_{n}\|u\| \\
& +\left(1-\mu_{n}\right)\left\|t_{n}-u\right\|+\lambda_{n}\left(1-\mu_{n}\right) \alpha_{n}\|u\| \\
& \leq\left\|x_{n}-u\right\|+\left\|y_{n}-u\right\|+2 \lambda_{n} \alpha_{n}\|u\|+\left(1-\mu_{n}\right)\left\|t_{n}-u\right\|,
\end{aligned}
$$

which hence implies that

$$
\begin{aligned}
\| t_{n} & -u \| \\
& \leq \frac{1}{\mu_{n}}\left[\left\|x_{n}-u\right\|+\left\|y_{n}-u\right\|+2 \lambda_{n} \alpha_{n}\|u\|\right] \\
& \leq \frac{1}{\mu_{n}}\left\{\left\|x_{n}-u\right\|+\frac{1}{\mu_{n}}\left(\left\|x_{n}-u\right\|+\lambda_{n} \alpha_{n}\|u\|\right)+2 \lambda_{n} \alpha_{n}\|u\|\right\} \\
& =\left(\frac{1}{\mu_{n}}+\frac{1}{\mu_{n}^{2}}\right)\left\|x_{n}-u\right\|+\left(\frac{1}{\mu_{n}^{2}}+\frac{2}{\mu_{n}}\right) \lambda_{n} \alpha_{n}\|u\| \\
& \leq \frac{1+2 \mu_{n}}{\mu_{n}^{2}}\left(\left\|x_{n}-u\right\|+\lambda_{n} \alpha_{n}\|u\|\right) .
\end{aligned}
$$

Thus, from (64) and (66), it follows that

$$
\begin{aligned}
\left\|y_{n}-u\right\|+\left\|t_{n}-u\right\| \leq & \frac{1}{\mu_{n}}\left(\left\|x_{n}-u\right\|+\lambda_{n} \alpha_{n}\|u\|\right) \\
& +\frac{1+2 \mu_{n}}{\mu_{n}^{2}}\left(\left\|x_{n}-u\right\|+\lambda_{n} \alpha_{n}\|u\|\right) \\
= & \frac{1+3 \mu_{n}}{\mu_{n}^{2}}\left(\left\|x_{n}-u\right\|+\lambda_{n} \alpha_{n}\|u\|\right) \\
\leq & \frac{4}{\mu_{n}^{2}}\left(\left\|x_{n}-u\right\|+\lambda_{n} \alpha_{n}\|u\|\right),
\end{aligned}
$$

which hence implies that

$$
\begin{aligned}
\left(\left\|y_{n}-u\right\|+\left\|t_{n}-u\right\|\right)^{2} & \leq \frac{16}{\mu_{n}^{4}}\left(\left\|x_{n}-u\right\|+\lambda_{n} \alpha_{n}\|u\|\right)^{2} \\
& \leq \frac{16}{\mu_{n}^{4}}\left(2\left\|x_{n}-u\right\|^{2}+2 \lambda_{n}^{2} \alpha_{n}^{2}\|u\|^{2}\right) \\
& \leq \frac{16}{\mu_{n}^{4}}\left(2\left\|x_{n}-u\right\|^{2}+2 \alpha_{n}^{2}\|u\|^{2}\right) \\
& \leq \frac{32}{\mu_{n}^{4}}\left\|x_{n}-u\right\|^{2}+\frac{32}{\mu_{n}^{4}} \alpha_{n}^{2}\|u\|^{2} .
\end{aligned}
$$

Repeating the same arguments as in (33) and (34), we can obtain that

$$
\begin{aligned}
\| t_{n}- & u \|^{2} \\
\leq & \left\|x_{n}-u\right\|^{2}+\left(\lambda_{n}^{2}\left(\alpha_{n}+L\right)^{2}-1\right)\left\|x_{n}-y_{n}\right\|^{2} \\
& +2 \lambda_{n} \alpha_{n}\|u\|\left(\left\|y_{n}-u\right\|+\left\|t_{n}-u\right\|\right) \\
\leq & \left\|x_{n}-u\right\|^{2}+2 \lambda_{n} \alpha_{n}\|u\|\left(\left\|y_{n}-u\right\|+\left\|t_{n}-u\right\|\right), \\
\| z_{n}- & u \|^{2} \\
\leq & \left(1+\gamma_{n}\right)\left\|t_{n}-u\right\|^{2}+\sigma_{n}\left\|Q t_{n}-u\right\|^{2} \\
& +\beta_{n}\left(\kappa-\left(1-\sigma_{n}-\beta_{n}\right)\right)\left\|t_{n}-S^{n} t_{n}\right\|^{2}+c_{n} \\
\leq & \left(1+\gamma_{n}\right)\left\|t_{n}-u\right\|^{2}+\sigma_{n}\left\|Q t_{n}-u\right\|^{2}+c_{n} \\
\leq & \left(1+\gamma_{n}\right)\left[\left\|x_{n}-u\right\|^{2}+2 \lambda_{n} \alpha_{n}\|u\|\left(\left\|y_{n}-u\right\|+\left\|t_{n}-u\right\|\right)\right] \\
& +\sigma_{n}\left\|Q t_{n}-u\right\|^{2}+c_{n} \\
= & \left(1+\gamma_{n}\right)\left\|x_{n}-u\right\|^{2}+2 \alpha_{n} \lambda_{n}\left(1+\gamma_{n}\right)\|u\| \\
& \times\left(\left\|y_{n}-u\right\|+\left\|t_{n}-u\right\|\right)+\sigma_{n}\left\|Q t_{n}-u\right\|^{2}+c_{n}
\end{aligned}
$$




$$
\begin{aligned}
& \leq\left(1+\gamma_{n}\right)\left\|x_{n}-u\right\|^{2} \\
& +\alpha_{n}\left[\lambda_{n}^{2}\left(1+\gamma_{n}\right)^{2}\|u\|^{2}+\left(\left\|y_{n}-u\right\|+\left\|t_{n}-u\right\|\right)^{2}\right] \\
& +\sigma_{n}\left(\left\|Q t_{n}-Q u\right\|+\|Q u-u\|\right)^{2}+c_{n} \\
& \leq\left(1+\gamma_{n}\right)\left\|x_{n}-u\right\|^{2} \\
& +\alpha_{n}\left[\left(1+\gamma_{n}\right)^{2}\|u\|^{2}+\left(\left\|y_{n}-u\right\|+\left\|t_{n}-u\right\|\right)^{2}\right] \\
& +\sigma_{n}\left[\rho\left\|t_{n}-u\right\|+(1-\rho) \frac{1}{1-\rho}\|Q u-u\|\right]^{2}+c_{n} \\
& \leq\left(1+\gamma_{n}\right)\left\|x_{n}-u\right\|^{2} \\
& +\alpha_{n}\left[\left(1+\gamma_{n}\right)^{2}\|u\|^{2}+\left(\left\|y_{n}-u\right\|+\left\|t_{n}-u\right\|\right)^{2}\right] \\
& +\sigma_{n}\left[\rho\left\|t_{n}-u\right\|^{2}+(1-\rho) \frac{1}{(1-\rho)^{2}}\|Q u-u\|^{2}\right]+c_{n} \\
& \leq\left\|x_{n}-u\right\|^{2}+\gamma_{n}\left\|x_{n}-u\right\|^{2}+\alpha_{n}\left(1+\gamma_{n}\right)^{2}\|u\|^{2} \\
& +\left(\alpha_{n}+\gamma_{n}\right)\left(\left\|y_{n}-u\right\|+\left\|t_{n}-u\right\|\right)^{2} \\
& +\sigma_{n}\left\|t_{n}-u\right\|^{2}+\sigma_{n}(1-\rho)^{-1}\|\mathrm{Qu}-u\|^{2}+c_{n} \\
& \leq\left\|x_{n}-u\right\|^{2}+\left(\alpha_{n}+\gamma_{n}\right)\left\|x_{n}-u\right\|^{2}+\alpha_{n}\left(1+\gamma_{n}\right)^{2}\|u\|^{2} \\
& +\left(\alpha_{n}+\gamma_{n}\right)\left(\left\|y_{n}-u\right\|+\left\|t_{n}-u\right\|\right)^{2} \\
& +\sigma_{n}\left(\left\|y_{n}-u\right\|+\left\|t_{n}-u\right\|\right)^{2} \\
& +\sigma_{n}\left((1-\rho)^{-1}+\gamma_{n}\right)\|Q u-u\|^{2}+c_{n} \\
& \leq\left\|x_{n}-u\right\|^{2}+\left(\alpha_{n}+\gamma_{n}\right)\left\|x_{n}-u\right\|^{2} \\
& +\alpha_{n}\left((1-\rho)^{-1}+\gamma_{n}\right)^{2}\|u\|^{2} \\
& +\left(\alpha_{n}+\gamma_{n}+\sigma_{n}\right)\left(\left\|y_{n}-u\right\|+\left\|t_{n}-u\right\|\right)^{2} \\
& +\sigma_{n}\left((1-\rho)^{-1}+\gamma_{n}\right)\|Q u-u\|^{2}+c_{n} \\
& \leq\left\|x_{n}-u\right\|^{2} \\
& +\left(\alpha_{n}+\gamma_{n}+\sigma_{n}\right)\left[\left\|x_{n}-u\right\|^{2}+\left(\left\|y_{n}-u\right\|+\left\|t_{n}-u\right\|\right)^{2}\right] \\
& +\alpha_{n}\left((1-\rho)^{-1}+\gamma_{n}\right)^{2}\|u\|^{2} \\
& +\sigma_{n}\left((1-\rho)^{-1}+\gamma_{n}\right)\|Q u-u\|^{2}+c_{n} \\
& \leq\left\|x_{n}-u\right\|^{2}+\left(\alpha_{n}+\gamma_{n}+\sigma_{n}\right) \\
& \times\left[\left\|x_{n}-u\right\|^{2}+\left(\left\|y_{n}-u\right\|+\left\|t_{n}-u\right\|\right)^{2}\right] \\
& +\left(\alpha_{n}+\gamma_{n}+\sigma_{n}\right)\left((1-\rho)^{-1}+\gamma_{n}\right)^{2}\left(\|u\|^{2}+\|Q u-u\|^{2}\right) \\
& +c_{n}
\end{aligned}
$$

$$
\begin{aligned}
= & \left\|x_{n}-u\right\|^{2}+\left(\alpha_{n}+\gamma_{n}+\sigma_{n}\right) \\
\times & \left\{\left\|x_{n}-u\right\|^{2}+\left(\left\|y_{n}-u\right\|+\left\|t_{n}-u\right\|\right)^{2}\right. \\
& \left.+\left((1-\rho)^{-1}+\gamma_{n}\right)^{2}\left(\|u\|^{2}+\|Q u-u\|^{2}\right)\right\}+c_{n} \\
\leq & \left\|x_{n}-u\right\|^{2}+\left(\alpha_{n}+\gamma_{n}+\sigma_{n}\right) \\
& \times\left\{\left\|x_{n}-u\right\|^{2}+\frac{32}{\mu_{n}^{4}}\left\|x_{n}-u\right\|^{2}+\frac{32}{\mu_{n}^{4}} \alpha_{n}^{2}\|u\|^{2}\right. \\
& \left.+\left((1-\rho)^{-1}+\gamma_{n}\right)^{2}\left(\|u\|^{2}+\|Q u-u\|^{2}\right)\right\}+c_{n} \\
= & \left\|x_{n}-u\right\|^{2}+\left(\alpha_{n}+\gamma_{n}+\sigma_{n}\right) \\
& \times\left\{\frac{32+\mu_{n}^{4}}{\mu_{n}^{4}}\left\|x_{n}-u\right\|^{2}+\left[\left((1-\rho)^{-1}+\gamma_{n}\right)^{2}+\frac{32}{\mu_{n}^{4}} \alpha_{n}^{2}\right]\right. \\
& \left.\times\|u\|^{2}+\left((1-\rho)^{-1}+\gamma_{n}\right)^{2}\|Q u-u\|^{2}\right\}+c_{n} \\
\leq & \left\|x_{n}-u\right\|^{2}+\left(\alpha_{n}+\gamma_{n}+\sigma_{n}\right) \\
& \times\left\{\frac{33}{\mu_{n}^{4}}\left\|x_{n}-u\right\|^{2}+\left[\left((1-\rho)^{-1}+\gamma_{n}\right)^{2}+\frac{32}{\mu_{n}^{4}} \alpha_{n}^{2}\right]\right. \\
& \left.\times\left(\|u\|^{2}+\|Q u-u\|^{2}\right)\right\}+c_{n} \\
\leq & x_{n}-u \|^{2}+c_{n}+\left(\alpha_{n}+\gamma_{n}+\sigma_{n}\right) \Delta_{n},
\end{aligned}
$$

for every $n=1,2, \ldots$ and hence $u \in C_{n}$. So, $\operatorname{Fix}(S) \cap \Gamma \subset$ $C_{n}$ for every $n=1,2, \ldots$ Next, let us show by mathematical induction that $\left\{x_{n}\right\}$ is well-defined and $\operatorname{Fix}(S) \cap \Gamma \subset C_{n} \cap Q_{n}$ for every $n=1,2, \ldots$. For $n=1$, we have $Q_{1}=C$. Hence, we obtain $\operatorname{Fix}(S) \cap \Gamma \subset C_{1} \cap Q_{1}$. Suppose that $x_{k}$ is given and $\operatorname{Fix}(S) \cap \Gamma \subset C_{k} \cap Q_{k}$ for some integer $k \geq 1$. Since $\operatorname{Fix}(S) \cap \Gamma$ is nonempty, $C_{k} \cap Q_{k}$ is a nonempty closed convex subset of $C$. So, there exists a unique element $x_{k+1} \in C_{k} \cap Q_{k}$ such that $x_{k+1}=P_{C_{k} \cap Q_{k}} x$. It is also obvious that there holds $\left\langle x_{k+1}-\right.$ $\left.z, x-x_{k+1}\right\rangle \geq 0$ for every $z \in C_{k} \cap Q_{k}$. Since $\operatorname{Fix}(S) \cap \Gamma \subset$ $C_{k} \cap Q_{k}$, we have $\left\langle x_{k+1}-z, x-x_{k+1}\right\rangle \geq 0$ for every $z \in \operatorname{Fix}(S) \cap \Gamma$ and hence $\operatorname{Fix}(S) \cap \Gamma \subset Q_{k+1}$. Therefore, we obtain $\operatorname{Fix}(S) \cap \Gamma \subset$ $C_{k+1} \cap Q_{k+1}$.

Step 2. $\lim _{n \rightarrow \infty}\left\|x_{n}-x_{n+1}\right\|=0$ and $\lim _{n \rightarrow \infty}\left\|x_{n}-z_{n}\right\|=0$.

Indeed, let $q=P_{\mathrm{Fix}(S) \cap \Gamma} x$. From $x_{n+1}=P_{\mathrm{C}_{n} \cap \mathrm{Q}_{n}} x$ and $q \epsilon$ $\operatorname{Fix}(S) \cap \Gamma \subset C_{n} \cap Q_{n}$, we have

$$
\left\|x_{n+1}-x\right\| \leq\|q-x\|
$$

for every $n=1,2, \ldots$ Therefore, $\left\{x_{n}\right\}$ is bounded. From (64), (66), and (70), we also obtain that $\left\{y_{n}\right\},\left\{t_{n}\right\}$, and $\left\{z_{n}\right\}$ are bounded. Since $x_{n+1} \in C_{n} \cap Q_{n} \subset Q_{n}$ and $x_{n}=P_{Q_{n}} x$, we have

$$
\left\|x_{n}-x\right\| \leq\left\|x_{n+1}-x\right\|
$$


for every $n=1,2, \ldots$. Therefore, there exists $\lim _{n \rightarrow \infty}\left\|x_{n}-x\right\|$. Since $x_{n}=P_{\mathrm{Q}_{n}} x$ and $x_{n+1} \in \mathrm{Q}_{n}$, using Proposition 4(ii), we have

$$
\left\|x_{n+1}-x_{n}\right\|^{2} \leq\left\|x_{n+1}-x\right\|^{2}-\left\|x_{n}-x\right\|^{2}
$$

for every $n=1,2, \ldots$ This implies that

$$
\lim _{n \rightarrow \infty}\left\|x_{n+1}-x_{n}\right\|=0
$$

Since $x_{n+1} \in C_{n}$, we have

$$
\left\|z_{n}-x_{n+1}\right\|^{2} \leq\left\|x_{n}-x_{n+1}\right\|^{2}+\theta_{n}
$$

which implies that

$$
\left\|z_{n}-x_{n+1}\right\| \leq\left\|x_{n}-x_{n+1}\right\|+\sqrt{\theta_{n}} .
$$

Hence, we get

$\left\|x_{n}-z_{n}\right\| \leq\left\|x_{n}-x_{n+1}\right\|+\left\|x_{n+1}-z_{n}\right\| \leq 2\left\|x_{n+1}-x_{n}\right\|+\sqrt{\theta_{n}}$

for every $n=1,2, \ldots$. From $\left\|x_{n+1}-x_{n}\right\| \rightarrow 0$ and $\theta_{n} \rightarrow 0$, we conclude that $\left\|x_{n}-z_{n}\right\| \rightarrow 0$ as $n \rightarrow \infty$.

Step 3. $\lim _{n \rightarrow \infty}\left\|x_{n}-y_{n}\right\|=0, \lim _{n \rightarrow \infty}\left\|x_{n}-t_{n}\right\|=0$ and $\lim _{n \rightarrow \infty}\left\|x_{n}-S x_{n}\right\|=0$.

Indeed, substituting (69) in (70), we get

$$
\begin{aligned}
& \left\|z_{n}-u\right\|^{2} \\
& \leq\left(1+\gamma_{n}\right)\left\|t_{n}-u\right\|^{2}+\sigma_{n}\left\|Q t_{n}-u\right\|^{2}+c_{n} \\
& \leq\left(1+\gamma_{n}\right)\left[\left\|x_{n}-u\right\|^{2}+\left(\lambda_{n}^{2}\left(\alpha_{n}+L\right)^{2}-1\right)\right. \\
& \times\left\|x_{n}-y_{n}\right\|^{2} \\
& \left.+2 \lambda_{n} \alpha_{n}\|u\|\left(\left\|y_{n}-u\right\|+\left\|t_{n}-u\right\|\right)\right] \\
& +\sigma_{n}\left\|Q t_{n}-u\right\|^{2}+c_{n} \\
& =\left\|x_{n}-u\right\|^{2}+\gamma_{n}\left\|x_{n}-u\right\|^{2}+\left(1+\gamma_{n}\right)\left(\lambda_{n}^{2}\left(\alpha_{n}+L\right)^{2}-1\right) \\
& \quad \times\left\|x_{n}-y_{n}\right\|^{2}+2 \alpha_{n} \lambda_{n}\left(1+\gamma_{n}\right)\|u\|\left(\left\|y_{n}-u\right\|+\left\|t_{n}-u\right\|\right) \\
& +\sigma_{n}\left\|Q t_{n}-u\right\|^{2}+c_{n},
\end{aligned}
$$

which hence implies that

$$
\begin{aligned}
(1+ & \left.\gamma_{n}\right)\left(b^{2}\left(\alpha_{n}+L\right)^{2}-1\right)\left\|x_{n}-y_{n}\right\|^{2} \\
\leq & \left(1+\gamma_{n}\right)\left(\lambda_{n}^{2}\left(\alpha_{n}+L\right)^{2}-1\right)\left\|x_{n}-y_{n}\right\|^{2} \\
\leq & \left\|x_{n}-u\right\|^{2}-\left\|z_{n}-u\right\|^{2}+\gamma_{n}\left\|x_{n}-u\right\|^{2} \\
& +2 \alpha_{n} \lambda_{n}\left(1+\gamma_{n}\right)\|u\|\left(\left\|y_{n}-u\right\|+\left\|t_{n}-u\right\|\right) \\
& +\sigma_{n}\left\|Q t_{n}-u\right\|^{2}+c_{n} \\
\leq & \left(\left\|x_{n}-u\right\|+\left\|z_{n}-u\right\|\right)\left\|x_{n}-z_{n}\right\|+\gamma_{n}\left\|x_{n}-u\right\|^{2} \\
& +2 \alpha_{n} \lambda_{n}\left(1+\gamma_{n}\right)\|u\|\left(\left\|y_{n}-u\right\|+\left\|t_{n}-u\right\|\right) \\
& +\sigma_{n}\left\|Q t_{n}-u\right\|^{2}+c_{n} .
\end{aligned}
$$

Since $\left\|x_{n}-z_{n}\right\| \rightarrow 0, \alpha_{n} \rightarrow 0, \gamma_{n} \rightarrow 0, \sigma_{n} \rightarrow 0$, and $c_{n} \rightarrow 0$, from the boundedness of $\left\{x_{n}\right\},\left\{y_{n}\right\},\left\{t_{n}\right\}$, and $\left\{z_{n}\right\}$, it follows that

$$
\lim _{n \rightarrow \infty}\left\|x_{n}-y_{n}\right\|=0 .
$$

Meantime, utilizing the arguments similar to those in (69), we have

$$
\left\|t_{n}-u\right\|^{2}
$$

$$
\begin{aligned}
\leq & \left\|x_{n}-u\right\|^{2}-\left\|x_{n}-y_{n}\right\|^{2}-\left\|y_{n}-t_{n}\right\|^{2} \\
& +2 \lambda_{n}\left(\alpha_{n}+L\right)\left\|x_{n}-y_{n}\right\|\left\|t_{n}-y_{n}\right\| \\
& +2 \lambda_{n} \alpha_{n}\|u\|\left(\left\|y_{n}-u\right\|+\left\|t_{n}-u\right\|\right) \\
\leq & \left\|x_{n}-u\right\|^{2}-\left\|x_{n}-y_{n}\right\|^{2}-\left\|y_{n}-t_{n}\right\|^{2} \\
& +\lambda_{n}^{2}\left(\alpha_{n}+L\right)^{2}\left\|t_{n}-y_{n}\right\|^{2}+\left\|x_{n}-y_{n}\right\|^{2} \\
& +2 \lambda_{n} \alpha_{n}\|u\|\left(\left\|y_{n}-u\right\|+\left\|t_{n}-u\right\|\right) \\
= & \left\|x_{n}-u\right\|^{2}+\left(\lambda_{n}^{2}\left(\alpha_{n}+L\right)^{2}-1\right)\left\|t_{n}-y_{n}\right\|^{2} \\
& +2 \lambda_{n} \alpha_{n}\|u\|\left(\left\|y_{n}-u\right\|+\left\|t_{n}-u\right\|\right) .
\end{aligned}
$$

Substituting (81) in (70), we get

$$
\begin{aligned}
& \left\|z_{n}-u\right\|^{2} \\
& \leq\left(1+\gamma_{n}\right)\left\|t_{n}-u\right\|^{2}+\sigma_{n}\left\|Q t_{n}-u\right\|^{2}+c_{n} \\
& \leq\left(1+\gamma_{n}\right)\left[\left\|x_{n}-u\right\|^{2}+\left(\lambda_{n}^{2}\left(\alpha_{n}+L\right)^{2}-1\right)\left\|t_{n}-y_{n}\right\|^{2}\right. \\
& \left.\quad+2 \lambda_{n} \alpha_{n}\|u\|\left(\left\|y_{n}-u\right\|+\left\|t_{n}-u\right\|\right)\right] \\
& \quad+\sigma_{n}\left\|Q t_{n}-u\right\|^{2}+c_{n} \\
& =\left\|x_{n}-u\right\|^{2}+\gamma_{n}\left\|x_{n}-u\right\|^{2}+\left(1+\gamma_{n}\right)\left(\lambda_{n}^{2}\left(\alpha_{n}+L\right)^{2}-1\right) \\
& \quad \times\left\|t_{n}-y_{n}\right\|^{2}+2 \alpha_{n} \lambda_{n}\left(1+\gamma_{n}\right)\|u\|\left(\left\|y_{n}-u\right\|+\left\|t_{n}-u\right\|\right) \\
& \quad+\sigma_{n}\left\|Q t_{n}-u\right\|^{2}+c_{n},
\end{aligned}
$$


which hence implies that

$$
\begin{aligned}
(1+ & \left.\gamma_{n}\right)\left(b^{2}\left(\alpha_{n}+L\right)^{2}-1\right)\left\|t_{n}-y_{n}\right\|^{2} \\
\leq & \left(1+\gamma_{n}\right)\left(\lambda_{n}^{2}\left(\alpha_{n}+L\right)^{2}-1\right)\left\|t_{n}-y_{n}\right\|^{2} \\
\leq & \left\|x_{n}-u\right\|^{2}-\left\|z_{n}-u\right\|^{2}+\gamma_{n}\left\|x_{n}-u\right\|^{2} \\
& +2 \alpha_{n} \lambda_{n}\left(1+\gamma_{n}\right)\|u\|\left(\left\|y_{n}-u\right\|+\left\|t_{n}-u\right\|\right) \\
& +\sigma_{n}\left\|Q t_{n}-u\right\|^{2}+c_{n} \\
\leq & \left(\left\|x_{n}-u\right\|+\left\|z_{n}-u\right\|\right)\left\|x_{n}-z_{n}\right\|+\gamma_{n}\left\|x_{n}-u\right\|^{2} \\
& +2 \alpha_{n} \lambda_{n}\left(1+\gamma_{n}\right)\|u\|\left(\left\|y_{n}-u\right\|+\left\|t_{n}-u\right\|\right) \\
& +\sigma_{n}\left\|Q t_{n}-u\right\|^{2}+c_{n} .
\end{aligned}
$$

Since $\left\|x_{n}-z_{n}\right\| \rightarrow 0, \alpha_{n} \rightarrow 0, \gamma_{n} \rightarrow 0, \sigma_{n} \rightarrow 0$, and $c_{n} \rightarrow 0$, from the boundedness of $\left\{x_{n}\right\},\left\{y_{n}\right\},\left\{t_{n}\right\}$, and $\left\{z_{n}\right\}$, it follows that

$$
\lim _{n \rightarrow \infty}\left\|t_{n}-y_{n}\right\|=0
$$

This together with $\left\|x_{n}-y_{n}\right\| \rightarrow 0$ implies that

$$
\lim _{n \rightarrow \infty}\left\|x_{n}-t_{n}\right\|=0
$$

Since $z_{n}=\left(1-\sigma_{n}-\beta_{n}\right) t_{n}+\sigma_{n} Q t_{n}+\beta_{n} S^{n} t_{n}$, we have $\beta_{n}\left(S^{n} t_{n}-t_{n}\right)=\left(z_{n}-t_{n}\right)-\sigma_{n}\left(Q t_{n}-t_{n}\right)$. Then,

$$
\begin{aligned}
\delta\left\|S^{n} t_{n}-t_{n}\right\| & \leq \beta_{n}\left\|S^{n} t_{n}-t_{n}\right\| \\
& \leq\left\|z_{n}-t_{n}\right\|+\sigma_{n}\left\|Q t_{n}-t_{n}\right\| \\
& \leq\left\|z_{n}-x_{n}\right\|+\left\|x_{n}-t_{n}\right\|+\sigma_{n}\left\|Q t_{n}-t_{n}\right\|
\end{aligned}
$$

and hence $\left\|t_{n}-S^{n} t_{n}\right\| \rightarrow 0$. Furthermore, observe that

$$
\left\|x_{n}-S^{n} x_{n}\right\| \leq\left\|x_{n}-t_{n}\right\|+\left\|t_{n}-S^{n} t_{n}\right\|+\left\|S^{n} t_{n}-S^{n} x_{n}\right\| .
$$

Utilizing Lemma 11, we have

$$
\begin{aligned}
& \left\|S^{n} t_{n}-S^{n} x_{n}\right\| \\
& \leq \frac{1}{1-\kappa}\left(\kappa\left\|t_{n}-x_{n}\right\|\right. \\
& \left.\quad+\sqrt{\left(1+(1-\kappa) \gamma_{n}\right)\left\|t_{n}-x_{n}\right\|^{2}+(1-\kappa) c_{n}}\right)
\end{aligned}
$$

for every $n=1,2, \ldots$. Hence, it follows from $\left\|x_{n}-t_{n}\right\| \rightarrow 0$ that $\left\|S^{n} t_{n}-S^{n} x_{n}\right\| \rightarrow 0$. Thus, from (87) and $\left\|t_{n}-S^{n} t_{n}\right\| \rightarrow$ 0 , we get $\left\|x_{n}-S^{n} x_{n}\right\| \rightarrow 0$. Since $\left\|x_{n+1}-x_{n}\right\| \rightarrow 0$,
$\left\|x_{n}-S^{n} x_{n}\right\| \rightarrow 0$ as $n \rightarrow \infty$, and $S$ is uniformly continuous, we obtain from Lemma 12 that $\left\|x_{n}-S x_{n}\right\| \rightarrow 0$ as $n \rightarrow \infty$.

Step 4. $\omega_{w}\left(x_{n}\right) \subset \operatorname{Fix}(S) \cap \Gamma$.

Indeed, repeating the same arguments as in Step 4 of the proof of Theorem 18, we can derive the desired conclusion.

Step 5. $\left\{x_{n}\right\},\left\{y_{n}\right\}$, and $\left\{z_{n}\right\}$ converge strongly to $q=P_{\mathrm{Fix}(S) \cap \Gamma} x$. Indeed, take $\widehat{u} \in \omega_{w}\left(x_{n}\right)$ arbitrarily. Then, $\widehat{u} \in \operatorname{Fix}(S) \cap$ $\Gamma$ according to Step 4. Moreover, there exists a subsequence $\left\{x_{n_{i}}\right\}$ of $\left\{x_{n}\right\}$ such that $x_{n_{i}} \rightarrow \widehat{u}$. Hence, from $q=P_{\operatorname{Fix}(S) \cap \Gamma} x$, $\widehat{u} \in \operatorname{Fix}(S) \cap \Gamma$, and (71), we have

$$
\begin{aligned}
\|q-x\| & \leq\|\widehat{u}-x\| \leq \liminf _{i \rightarrow \infty}\left\|x_{n_{i}}-x\right\| \\
& \leq \limsup _{i \rightarrow \infty}\left\|x_{n_{i}}-x\right\| \leq\|q-x\| .
\end{aligned}
$$

So, we obtain

$$
\lim _{i \rightarrow \infty}\left\|x_{n_{i}}-x\right\|=\|\widehat{u}-x\|
$$

From $x_{n_{i}}-x \rightarrow \widehat{u}-x$, we have $x_{n_{i}}-x \rightarrow \widehat{u}-x$ due to the Kadec-Klee property of Hilbert spaces [17]. So, it is clear that $x_{n_{i}} \rightarrow \widehat{u}$. Since $x_{n}=P_{Q_{n}} x$ and $q \in \operatorname{Fix}(S) \cap \Gamma \subset C_{n} \cap Q_{n} \subset Q_{n}$, we have

$$
\begin{aligned}
-\left\|q-x_{n_{i}}\right\|^{2} & =\left\langle q-x_{n_{i}}, x_{n_{i}}-x\right\rangle+\left\langle q-x_{n_{i}}, x-q\right\rangle \\
& \geq\left\langle q-x_{n_{i}}, x-q\right\rangle .
\end{aligned}
$$

As $i \rightarrow \infty$, we obtain $-\|q-\widehat{u}\|^{2} \geq\langle q-\widehat{u}, x-q\rangle \geq 0$ by $q=P_{\operatorname{Fix}(S) \cap \Gamma} x$ and $\widehat{u} \in \operatorname{Fix}(S) \cap \Gamma$. Hence, we have $\widehat{u}=q$. This implies that $x_{n} \rightarrow q$. It is easy to see that $y_{n} \rightarrow q$ and $z_{n} \rightarrow q$. This completes the proof.

\section{Acknowledgments}

In this research, first author was partially supported by the National Science Foundation of China (11071169), Innovation Program of Shanghai Municipal Education Commission (09ZZ133), and Ph.D. Profram Foundation of Ministry of Education of China (20123127110002). The research part of the second author was done during his visit to KFUPM, Dhahran, Saudi Arabia. The third author was partially supported by a Grant from the NSC 101-2115-M-037-001.

\section{References}

[1] K. Goebel and W. A. Kirk, "A fixed point theorem for asymptotically nonexpansive mappings," Proceedings of the American Mathematical Society, vol. 35, pp. 171-174, 1972.

[2] R. Bruck, T. Kuczumow, and S. Reich, "Convergence of iterates of asymptotically nonexpansive mappings in Banach spaces with the uniform Opial property," Colloquium Mathematicum, vol. 65 , no. 2, pp. 169-179, 1993.

[3] T.-H. Kim and H.-K. Xu, "Convergence of the modified Mann's iteration method for asymptotically strict pseudo-contractions," Nonlinear Analysis. Theory, Methods \& Applications, vol. 68, no. 9, pp. 2828-2836, 2008. 
[4] D. R. Sahu, H.-K. Xu, and J.-C. Yao, "Asymptotically strict pseudocontractive mappings in the intermediate sense," Nonlinear Analysis. Theory, Methods \& Applications, vol. 70, no. 10, pp. 3502-3511, 2009.

[5] L.-C. Ceng, Q. H. Ansari, and J.-C. Yao, "Some iterative methods for finding fixed points and for solving constrained convex minimization problems," Nonlinear Analysis. Theory, Methods \& Applications, vol. 74, no. 16, pp. 5286-5302, 2011.

[6] G. M. Korpelevič, "An extragradient method for finding saddle points and for other problems," Ėkonomika i Matematicheskie Metody, vol. 12, no. 4, pp. 747-756, 1976.

[7] R. P. Agarwal, D. O’Regan, and D. R. Sahu, “Iterative construction of fixed points of nearly asymptotically nonexpansive mappings," Journal of Nonlinear and Convex Analysis, vol. 8, no. 1, pp. 61-79, 2007.

[8] Q. H. Ansari, C. S. Lalitha, and M. Mehta, Generalized Convexity, Nonsmooth Variational Inequalities and Nonsmooth Optimization, Taylor \& Francis, Boca Raton, Fla, USA, 2013.

[9] A. Auslender and M. Teboulle, Asymptotic Cones and Functions in Optimization and Variational Inequalities, Springer Monographs in Mathematics, Springer, New York, NY, USA, 2003.

[10] F. Facchinei and J.-S. Pang, Finite-Dimensional Variational Inequalities and Complementarity Problems, vol. 1-2 of Springer Series in Operations Research, Springer, New York, NY, USA, 2003.

[11] R. Glowinski, Numerical Methods for Nonlinear Variational Problems, Springer Series in Computational Physics, Springer, New York, NY, USA, 1984.

[12] R. Glowinski, J.-L. Lions, and R. Trémolières, Numerical Analysis of Variational Inequalities, vol. 8 of Studies in Mathematics and its Applications, North-Holland Publishing, Amsterdam, The Netherlands, 1981.

[13] I. V. Konnov, Equilibrium Models and Variational Inequalities, vol. 210 of Mathematics in Science and Engineering, Elsevier B. V., Amsterdam, The Netherlands, 2007.

[14] J. Reinermann, "Über fixpunkte kontrahierender abbildungen und schwach konvergente Toeplitz-verfahren," Archiv der Mathematik, vol. 20, pp. 59-64, 1969.

[15] M. O. Osilike, S. C. Aniagbosor, and B. G. Akuchu, "Fixed points of asymptotically demicontractive mappings in arbitrary Banach spaces," Panamerican Mathematical Journal, vol. 12, no. 2, pp. 77-88, 2002.

[16] K.-K. Tan and H. K. Xu, "Approximating fixed points of nonexpansive mappings by the Ishikawa iteration process," Journal of Mathematical Analysis and Applications, vol. 178, no. 2, pp. 301-308, 1993.

[17] K. Goebel and W. A. Kirk, Topics in Metric Fixed Point Theory, vol. 28 of Cambridge Studies in Advanced Mathematics, Cambridge University Press, Cambridge, UK, 1990.

[18] Z. Opial, "Weak convergence of the sequence of successive approximations for nonexpansive mappings," Bulletin of the American Mathematical Society, vol. 73, pp. 591-597, 1967.

[19] R. T. Rockafellar, "On the maximality of sums of nonlinear monotone operators," Transactions of the American Mathematical Society, vol. 149, pp. 75-88, 1970.

[20] L.-C. Ceng and J.-C. Yao, "Strong convergence theorems for variational inequalities and fixed point problems of asymptotically strict pseudocontractive mappings in the intermediate sense," Acta Applicandae Mathematicae, vol. 115, no. 2, pp. 167191, 2011. 


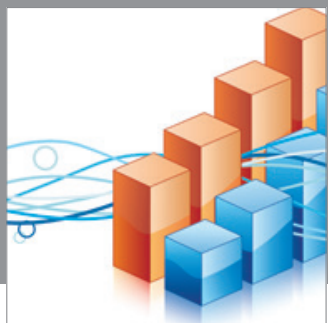

Advances in

Operations Research

mansans

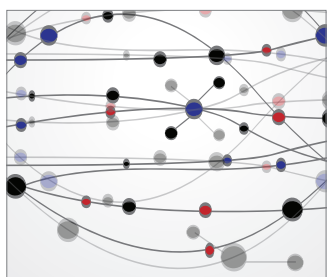

The Scientific World Journal
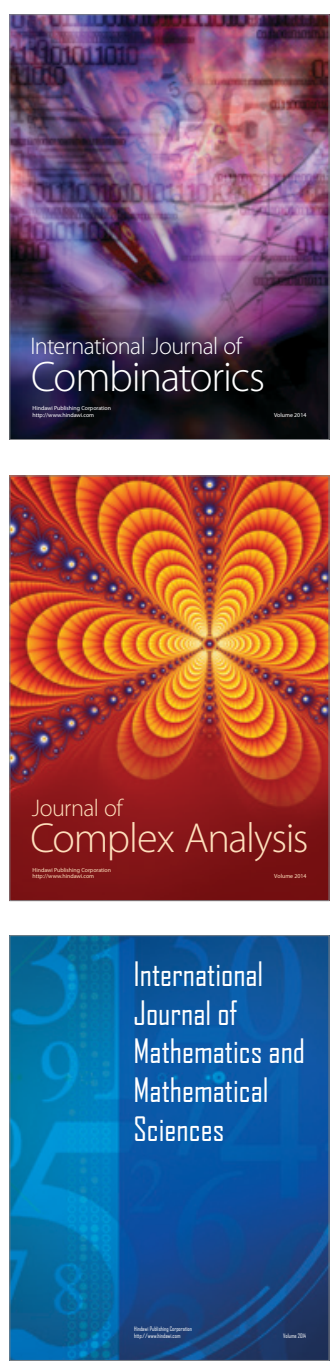
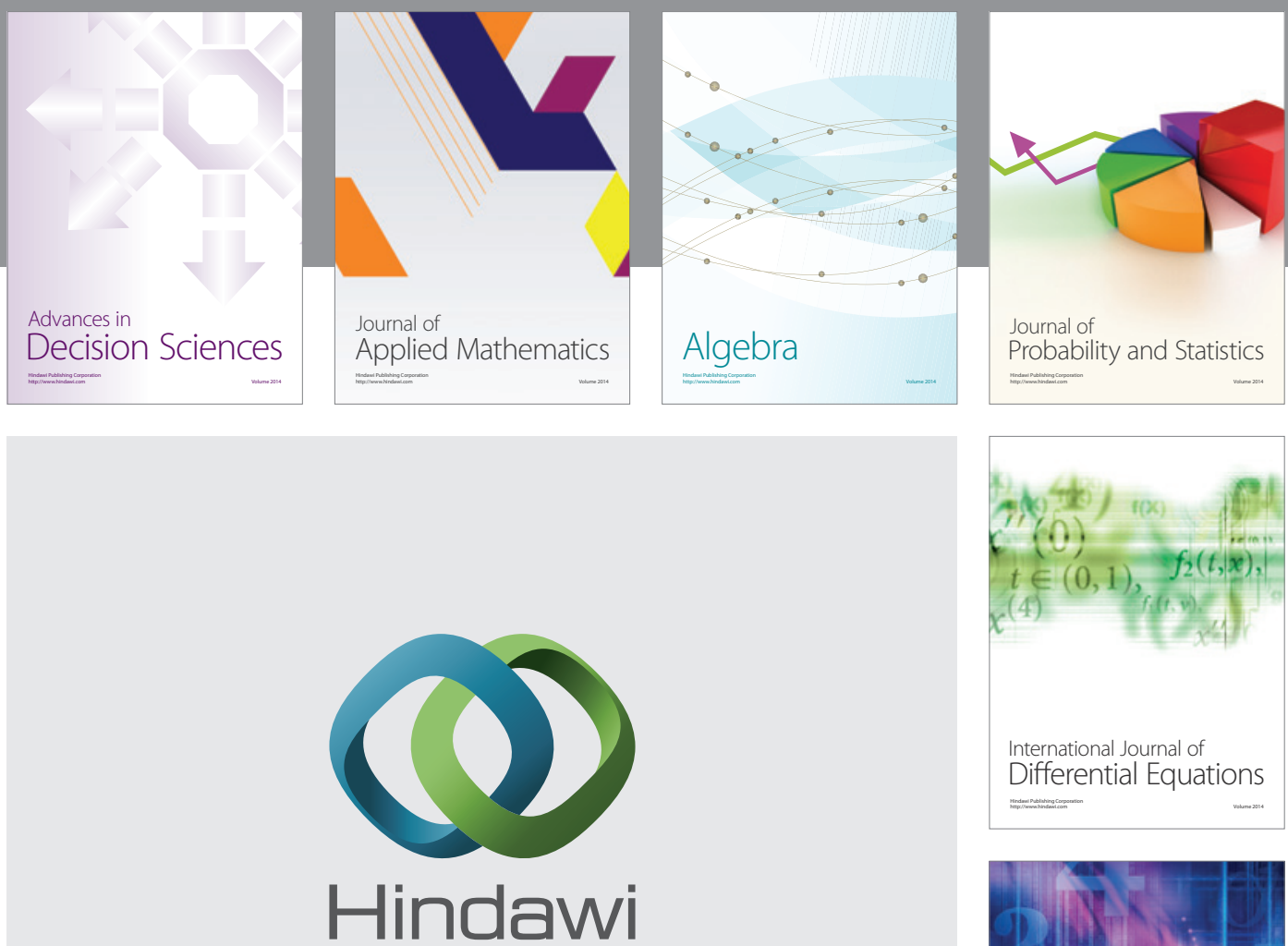

Submit your manuscripts at http://www.hindawi.com
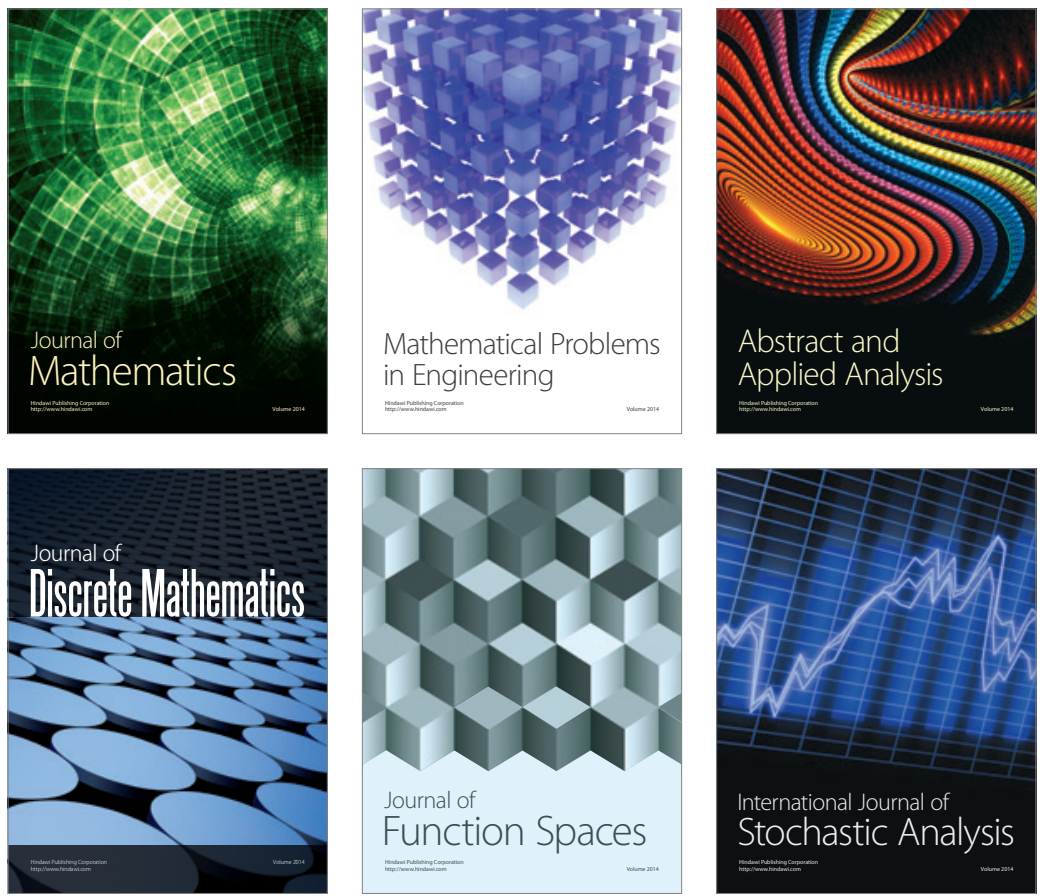

Journal of

Function Spaces

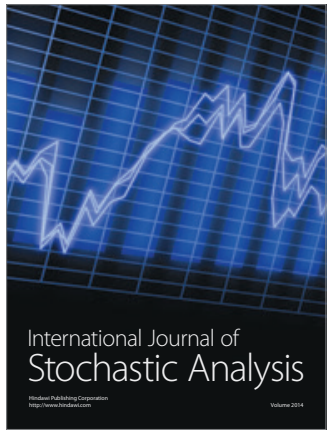

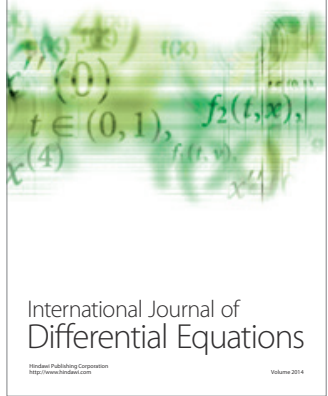
